\title{
Effect of Estradiol in an Azoxymethane/Dextran Sulfate Sodium-Treated Mouse Model of Colorectal Cancer: Implication for Sex Difference in Colorectal Cancer Development
}

\author{
Hee Jin Son, MSc ${ }^{1}$ \\ Sung Hwa Sohn, PhD² \\ Nayoung Kim, MD, PhD 23 \\ Ha-Na Lee, $\mathrm{PhD}^{4}$ \\ Sun Min Lee, $\mathrm{PhD}^{2}$ \\ Ryoung Hee Nam, MSc² \\ Ji Hyun Park, MSc ${ }^{3}$ \\ Chin-Hee Song, $\mathrm{PhD}^{2}$ \\ Eun Shin, MD, PhD ${ }^{5}$ \\ Hee Young Na, MSc ${ }^{5}$ \\ Joo Sung Kim, MD, $P h D^{3}$ \\ Dong Ho Lee, MD, PhD2,3 \\ Young-Joon Surh, $\mathrm{PhD}^{4}$
}

${ }^{1}$ Seoul National University College of Medicine, Seoul, ${ }^{2}$ Department of Internal Medicine, Seoul National University Bundang Hospital, Seongnam, ${ }^{3}$ Department of Internal Medicine and Liver Research Institute, Seoul National University College of Medicine, Seoul, ${ }^{4}$ Tumor Microenvironment Global Core Research Center, Seoul National University College of Pharmacy, Seoul, ${ }^{5}$ Department of Pathology, Seoul National University Bundang Hospital, Seongnam, Korea
Correspondence: Nayoung Kim, MD, PhD Department of Internal Medicine, Seoul National University Bundang Hospital, 82 Gumi-ro, 173 beon-gil, Bundang-gu, Seongnam 13620, Korea

Tel: 82-31-787-7008

Fax: 82-31-787-4051

E-mail: nayoungkim49@empas.com

Received January 23, 2018

Accepted July 20, 2018

Published Online August 1, 2018

*Hee Jin Son and Sung Hwa Sohn contributed equally to this work.

\section{Purpose}

This study demonstrates that estradiol downregulates inflammation and inhibits colorectal cancer (CRC) development in azoxymethane/dextran sulfate sodium (AOM/DSS) mouse model.

\section{Materials and Methods}

AOM/DSS-treated male and female mice were sacrificed at weeks 2, 10, and 16, to assess estrogen effects on colitis and carcinogenesis. Macroscopic and histologic severity of colitis and Western blot and quantitative real-time polymerase chain reaction were evaluated, to measure inflammatory mediators and cytokines.

\section{Results}

Compared with AOM/DSS-treated male mice (M-AOM/DSS group), AOM/DSS-treated male mice with estradiol administration (M-AOM/DSS+estr group) displayed at week 2 significantly decreased severity of colitis. At weeks 10 and 16, AOM/DSS-treated female mice (F-AOM/DSS group) and the M-AOM/DSS+estr group showed significantly lower tumor multiplicity compared with the M-AOM/DSS group. At week 2, F-AOM/DSS group had a lower level of nuclear factor-kB (NF-kB) expression and higher level of nuclear factor erythroid 2-related factor 2 (Nrf2) expression, compared to the M-AOM/DSS group. At week 2, expression levels of NF-KB and its related mediators decreased in the M-AOM/DSS+estr group, while levels of Nrf2 and Nrf2-related anti-oxidant enzymes increased. In addition, estradiol significantly increased Nod-like receptor protein 3 (NLRP3) inflammasome expressions in AOM/DSS-treated male mice. In contrast, at weeks 10 and 16, Nrf2 and its-related anti-oxidant enzymes and NLRP3 inflammasome were highly expressed in M-AOM/DSS group and in F-AOM/DSS group, who developed cancer.

\section{Conclusion}

The data suggest that estradiol inhibits the initiation of CRC by regulating Nrf2-related pathways. Moreover, these imply the dual role of Nrf2 and NLRP3 inflammasome, including promotion of tumor progression upon tumor initiation. 


\section{Introduction}

The incidence rate of colorectal cancer (CRC) is high in males compared with females, regardless of age, ethnicity, and geographic regions [1]. Epidemiologic studies have shown an obvious decrement of CRC incidence with oral contraceptive use [2], and lowered risk of CRC with estradiol plus progestin therapy [3]. Some preclinical research has yielded conflicting results on the influence of female sex hormones on CRC. That is, in ovariectomized female C57BL / 6J mice with a germ-line APC gene mutation, intestinal adenomas were reportedly increased by $77 \%(\mathrm{p}<0.05)$ compared to non-ovariectomized females, while supplementation of $17 \beta$-estradiol to ovariectomized female C57BL/6J mice reduced the number of adenomas to the same level as nonovariectomized mice [4]. However, in an $A P C^{\text {Pircl+ }}$ rat model, ovariectomized rats did not develop a higher prevalence of adenomas, while orchidectomy protected against colonic tumorigenesis [5]. In our previous study, we suggested the protective roles of estradiol in colorectal tumorigenesis by showing more tumor multiplicities in azoxymethane and dextran sulphate sodium (AOM/DSS)-treated male mice compared to AOM/DSS-treated female mice [6].

Estradiol increased nuclear factor erythroid 2-related factor 2 (Nrf2) activity in breast cancer cell line [7]. Yet in CRC, there is no comprehensive knowledge about estradiol as an upstream regulator of Nrf2. Protein kinase $\mathrm{C} \delta(\mathrm{PKC} \delta$ ), an important mediator in the $\mathrm{G}_{13}$ signaling pathway, promotes Nrf2 activity [8]. In addition, PKC has been closely related to the protective effect of estradiol on vascular reactivity after shock in female rats [9]. Furthermore, estradiol-induced protein synthesis in mouse uterine epithelial cells was also mediated through the PKC signaling pathway [10]. Estradiol increased the mRNA level of PKC $\delta$ in the colonic epithelium of rats [11]. Thus, the $\mathrm{G}_{13}-\mathrm{PKC} \delta$ signaling pathway could be an upstream regulator of Nrf2 in CRC, and estradiol might play a role in this cascade.

The enhancement of colitis-associated CRC development in Nrf2-deficient mice treated with AOM/DSS [12] supports the protective influence of Nrf2 against colonic inflammation. There are several suggested mechanisms of the Nrf2mediated prevention of inflammation and tumorigenesis. First, the activation of Nrf2 and cross-talk between Nrf2 and nuclear factor- $\mathrm{\kappa B}$ (NF- $\mathrm{\kappa}$ ) downregulate pro-inflammatory signaling by suppressing NF- $\kappa B$ directly [13]. Second, Nrf2 is one of the most essential transcription factors that regulate the expression of anti-oxidant enzymes [14]. Lastly, the close relationship of Nrf2 with the activating mechanism of the Nod-like receptor protein 3 (NLRP3) inflammasome was recently reported [15]. Caspase-1 activated by NLRP3 inflammasome triggers pyroptosis [16], and pyroptosis might elicit an anti-cancer immune reaction [17].

Inflammation is an important factor in the pathophysiology of colitis-associated and sporadic CRC. For example, Saleiro et al. [18] demonstrated the higher levels of inflammatory cytokines and polyp development at weeks 9 and 16 in AOM/DSS-treated estrogen receptor $\beta$ (ER $\beta)$ knockout mice, compared to wild-type mice. However, almost no studies have thoroughly evaluated the early inflammation stage of tumorigenesis, since in most of the studies, the animals were sacrificed after adenoma formation.

From this background, we hypothesized that the observed sex difference in CRC incidence may be due to estradiolmediated down-regulation of inflammation, which might somehow affect the CRC cascade. To explore this hypothesis, we assessed the temporal role of Nrf2 in modulating inflammation and carcinogenesis through the regulation of the NF- $\mathrm{kB}$-mediated pro-inflammatory pathway, anti-oxidant enzymes, and the NLRP3 inflammasome.

\section{Materials and Methods}

\section{Animals}

Four-week-old male and female ICR mice (Orient Co., Ltd., Seoul, Korea) were housed in cages, and maintained at $23^{\circ} \mathrm{C}$ with a 12/12-hour light/ dark cycle under specific pathogenfree conditions.

\section{Experimental design}

Fig. 1A shows the experimental design. After 1 week of acclimatization, male and female mice were randomized into five groups ( $\mathrm{n}=20-36$ / group). Group 1 male control (M-con) mice were sacrificed at week $2(n=4)$, and weeks 10 and 16 ( $\mathrm{n}=6$ each). Group 2 comprised male mice treated with AOM/ DSS (M-AOM/DSS). The mice were sacrificed at week 2 $(\mathrm{n}=6)$, and at weeks 10 and 16 ( $\mathrm{n}=12$ each). Group $3 \mathrm{com}-$ prised AOM/DSS-treated male mice administered estradiol (M-AOM/DSS+estr). The mice were sacrificed at week 2 $(\mathrm{n}=6)$, and weeks 10 and 16 ( $\mathrm{n}=12$ each). Group 4 comprised female control mice (F-con). They were sacrificed at week 2 $(\mathrm{n}=4)$, and weeks 10 and 16 ( $\mathrm{n}=6$ each). Group 5 comprised AOM/DSS-treated female mice (F-AOM/DSS). They were sacrificed at week $2(n=6)$, and weeks 10 and 16 ( $n=12$ each). $\mathrm{AOM} / \mathrm{DSS}$-treated male and female mice were intraperitoneally injected with AOM $(10 \mathrm{mg} / \mathrm{kg}$; Sigma-Aldrich, St. Louis, MO) on day 0 in the experimental schedule. For induction of colitis, $2.5 \%(\mathrm{w} / \mathrm{v})$ DSS (MP Biomedicals, Aurora, $\mathrm{OH}$ ) was supplied in drinking water for 7 days, 1 


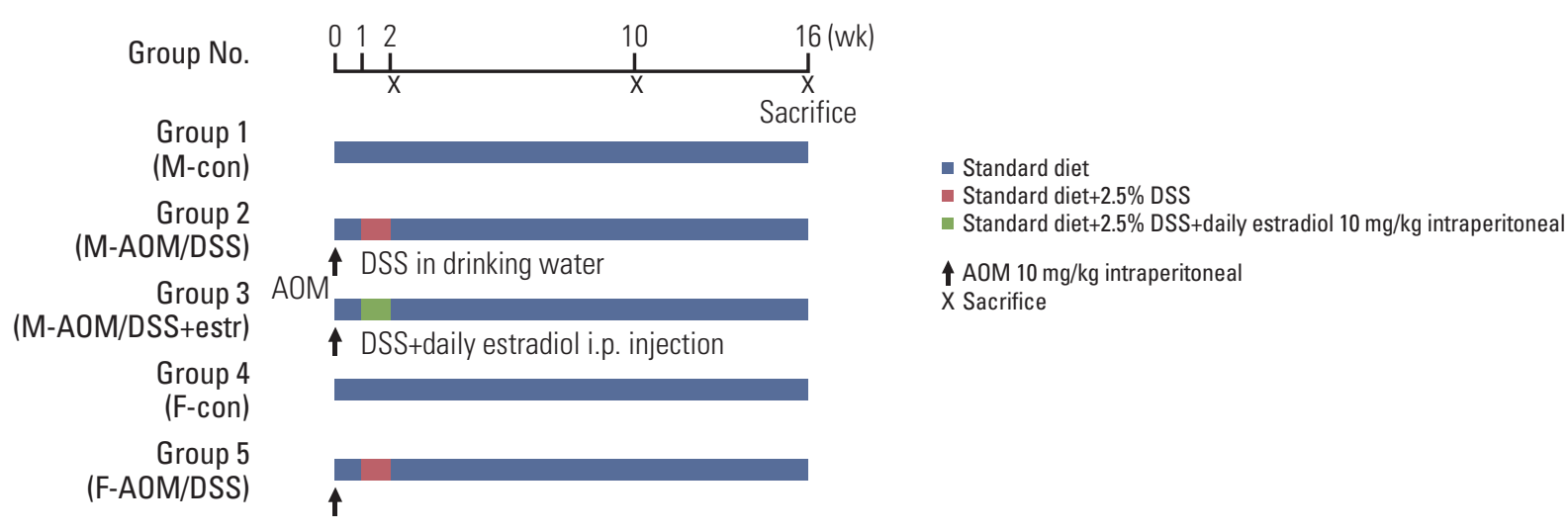

B

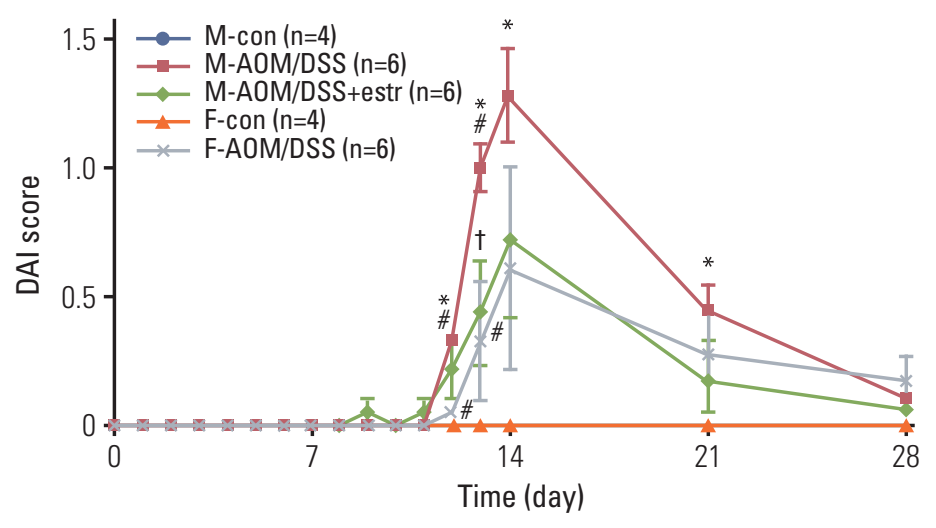

Week 2
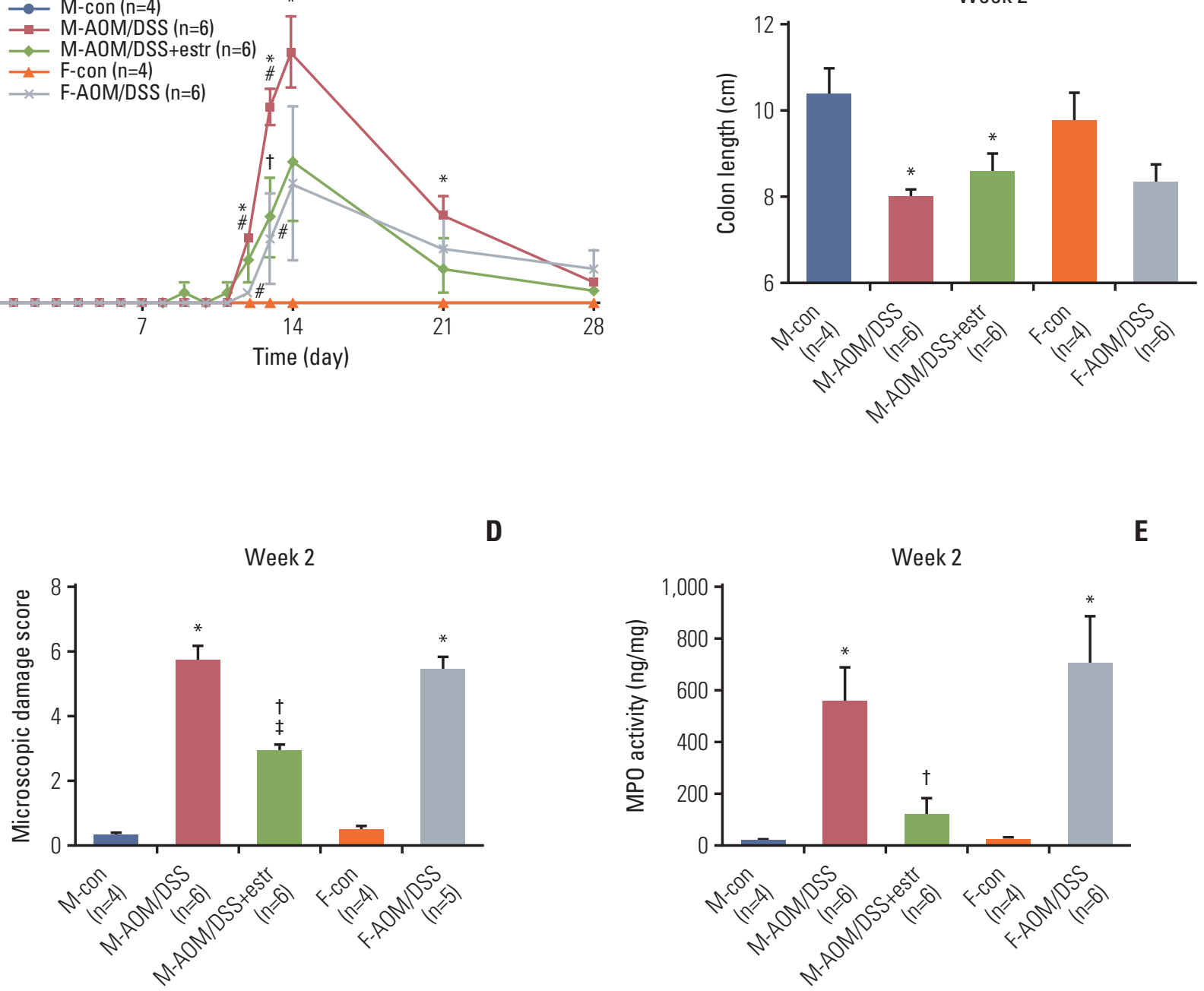

Fig. 1. Estradiol prevents wasting disease progression in azoxymethane/dextran sulfate sodium (AOM/DSS)-induced colitis. (A) Scheme for the experimental course of AOM/DSS promoted colitis-associated tumorigenesis. The mice were injected AOM on day 0. DSS in drinking water (2.5\%) and estradiol supply was provided from day 7 to 13 . Mice were sacrificed at week 2, 10, and 16. (B) Disease Activity Index (DAI) was decreased by estradiol. (C) Colon length at week 2. (D) Macroscopic damage score at week 2. (E) Myeloperoxidase (MPO) activity in colonic tissues at week 2. (Continued to the next page) 

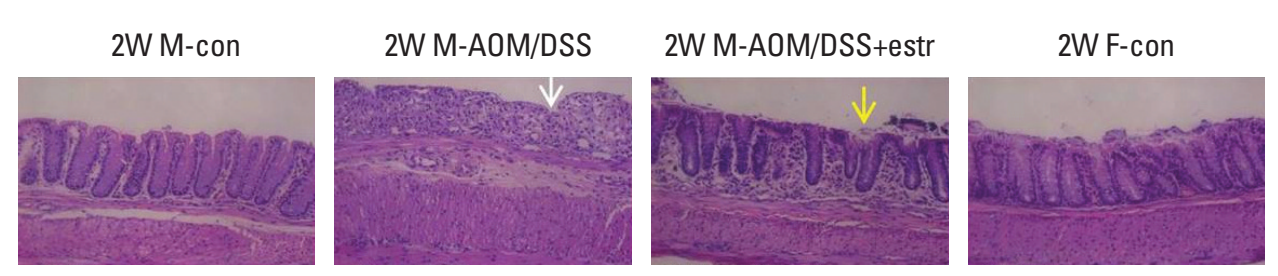

\section{W F-AOM/DSS}

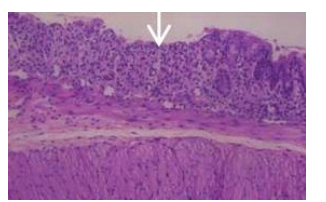

Fig. 1. (Continued from the previous page) (F) Histopathologic findings of the colonic mucosa (H\&E staining, $\times 200)$ at week 2 . In control mice, the mucosa is normal in males and females. However, near-total crypt loss and infiltration of severe inflammatory cell of colonic mucosa (white arrow) are seen in both males and females. Estradiol treatment significantly decreased histologic damage, with only mild erosion (yellow arrow). ${ }^{*} \mathrm{p}<0.05$ compared to control, ${ }^{+} \mathrm{p}<0.05$ compared to AOM/DSS group, ${ }^{\ddagger} \mathrm{p}<0.05$ between estradiol-treated group and female AOM/DSS group, ${ }^{\sharp} \mathrm{p}<0.05$ between the male AOM/DSS group and the female AOM/DSS group. M, male; F, female; estr, estradiol.

week following the injection of AOM [6]. The quantity of DSS consumed in the drinking water was checked on days 7, 9, and 11. M-AOM/DSS+estr mice were intraperitoneally injected each day for 7 days with $17 \beta$-estradiol $(10 \mathrm{mg} / \mathrm{kg}$; Sigma-Aldrich) dissolved in olive oil. The injections were done during the same period of DSS consumption. Animals were euthanized by $\mathrm{CO}_{2}$ asphyxiation at 2, 10, and 16 weeks after AOM injection (Fig. 1A).

\section{Evaluation of clinical symptoms}

Clinical symptoms were evaluated using the Disease Activity Index (DAI), which includes body weight loss, stool characterization, and hematochezia [6]. DAI was scored by two technicians (L.H.N. and D.C.) in a blinded manner.

\section{Lesion enumeration}

Colons extracted from cecum to the rectum were opened longitudinally, and stool was washed out with phosphatebuffered saline. Colon length was measured from cecum to rectum using a ruler. Polypoid lesions with a diameter $<2$ $\mathrm{mm}$ or $>2 \mathrm{~mm}$ were independently counted by three gastroenterologists in a blinded manner. Tumor multiplicity was defined as the number of gross polyps approved by the three gastroenterologists.

\section{Tissue processing, histopathology, and immunohisto- chemical analysis}

After extraction from the peritoneum, the colon was divided into proximal and distal portions. The proximal colon was half of the colon to $1.5 \mathrm{~cm}$ distal from the ileocecal valve. The distal colon was the other half up to the rectum $1.5 \mathrm{~cm}$ from the anal verge. One or two representative polyps of each sample were prepared for histological analysis. These samples were fixed with phosphate-buffered formalin, and stained with hematoxylin and eosin. Other portions were frozen in lipid nitrogen, and kept at $-70^{\circ} \mathrm{C}$, until use in the biochemical assays. The tumor incidence (\%) was determined as the percentage of rats having more than one tumor. The classification of adenoma and adenocarcinoma was performed as previously described [6]. The depth of invasion by adenocarcinoma in the colonic tissues was specified as mucosa or submucosa [6,19]. Their incidence was also measured.

Immunohistochemical (IHC) analysis of Nrf2 was performed. Tissue sections were treated with 3\% hydrogen peroxide, and nonspecific binding sites were blocked. The sections were incubated with anti-Nrf2 antibodies (ab31163, Abcam, Cambridge, MA). An automatic immunostainer (BenchMark XT, Ventana Medical Systems, Tucson, AZ) and UltraView Universial DAB detection kit (Ventana Medical Systems) were used for immunostaining. The proportion of the number of immune-stained in total cells of all crypts were calculated.

\section{Scoring of microscopic damage}

Histological severity was assessed using microscopic damage score reflecting colonic epithelial damage and depth of infiltration with inflammatory cells as previously described [6]. This was evaluated by a pathologist (E.S.) in a blinded manner.

\section{Measurement of inflammatory cytokines}

The levels of myeloperoxidase (MPO) in the colonic tissues were examined by ELISA (R\&D Systems, Minneapolis, MN). Every assay was performed in triplicate. 


\section{Western blot analysis}

Protein extracts were isolated using RIPA buffer (Cell Signaling Technology, Beverly, MA). Cytoplasmic and nuclear lysates were separated using a NE-PER Nuclear Cytoplasmic Extraction Reagent kit (Pierce, Rockford, IL), according to the manufacturer's instructions. Protein concentration was determined using the BCA protein assay reagent (Pierce). Protein samples were separated by 8 to $15 \%$ sodium dodecyl sulfate polyacrylamide gel electrophoresis. After blocking, membranes were incubated overnight at $4{ }^{\circ} \mathrm{C}$ with specific antibodies. S1 Table of the Supporting Information (SI) lists the primary antibodies in detail. Horseradish peroxidase-conjugated anti-rabbit, anti-goat, or anti-mouse immunoglobulin (Santa Cruz Biotechnology, Dallas, TX) was used as secondary antibodies.

\section{Quantitative real-time PCR analysis}

RNA was isolated from colon tissue using Trizol reagent (Invitrogen, Carlsbad, CA) according to the manufacturer's instruments, and quantified using a NanoDrop ND-1000 device (Thermo Scientific, Wilmington, DE). cDNA was synthesized using the High Capacity cDNA reverse Transcription Kit (Applied Biosystems, Foster City, CA). Quantitative real-time PCR was performed using Power SYBR Green PCR Master mix and a Viia7 instrument (Applied Biosystems). The transcript levels of glyceraldehydes-3-phosphate dehydrogenase were used for sample normalization. S2 Table of the SI lists the primer sequences.

\section{Statistical analyses}

Data are expressed as mean \pm standard error of mean. Statistical significance was examined by Mann-Whitney test or Fisher exact test. A p-value of $<0.05$ was considered to indicate statistical significance. All statistical analyses were conducted using SPSS ver. 18.0 (SPSS Inc., Chicago, IL) and GraphPad Prism software (GraphPad, La Jolla, CA).

\section{Ethical statement}

All animal experimental procedures were approved by the Institutional Animal Care and Use Committee (IACUC) of Seoul National University Bundang Hospital (BA1310-139/ 091-01). The procedures were in accordance with the Animals in Research: Reporting In Vivo Experiments (ARRIVE) statement.

\section{Results}

\section{Estradiol ameliorates histologic evidence of colonic inflammation by week 2}

We first analyzed DAI score, colon shortening, and severity of colitis, to evaluate the early impacts of estradiol. The M-AOM/DSS and F-AOM/DSS groups displayed higher DAI scores compared to the control mice (M-con and F-con groups), suggesting the induction of severe colitis $(\mathrm{p}=0.007$ at week 2 for M-AOM/DSS group vs. M-con group) (Fig. 1B). The M-AOM/DSS+estr group had lower DAI scores than the M-AOM/DSS group on day $13(\mathrm{p}=0.049)$, and at week 2 (Fig. 1B). Colon length of the M-AOM/DSS group was shortened by inflammation at week 2 (Fig. 1C). Representative histologic images (Fig. 1F) and microscopic damage score revealed significantly less infiltration of inflammatory cells and mild cryptic damage for the M-AOM/DSS+estr group compared to the M-AOM / DSS group at week 2 ( $\mathrm{p}=0.004$ for microscopic damage score) (Fig. 1D). The M-AOM/DSS+estr group displayed a lower level of $\mathrm{MPO}$, a mediator associated with intestinal inflammation, compared to the M-AOM/DSS group ( $p=0.020$ ) (Fig. 1E). The collective data indicate that estradiol reduced the severity of DSS-induced colitis.

\section{Estradiol attenuates colitis-associated, histology-evident tumorigenesis at weeks 10 and 16}

Prominent polyps developed at weeks 10 and 16, mostly in the distal part of the colon (Table 1, Fig. 2A and B), consistent with a previous report [20]. The development of polyps was obvious in the M-AOM/DSS group, while only a few polyps developed in the F-AOM/DSS group at week 10 ( $\mathrm{p}=0.014$ for tumor number) (Fig. 2A and C). The findings provided evidence of a significant sex difference in colitisassociated tumor development. An astonishing result was the absence of visible polyps in the M-AOM/DSS+estr group at week 10 (Fig. 2A). The sex difference was also present at week 16; the F-AOM/DSS group displayed fewer tumors and lower incidence of colonic neoplasms compared to the M-AOM / DSS group ( $\mathrm{p}=0.001$ for tumor incidence) (Table 1, Fig. 2B and D). In the F-AOM/DSS group, CRC had developed in four of $12(25 \%)$ of the mice at week 16 , which was significantly lower than the M-AOM/DSS group (Table 1). Polyps were observed in the M-AOM/DSS+estr group at week 16, but at markedly fewer numbers than the M-AOM/ DSS group ( $p=0.020$ ) (Fig. 2B). These data were evidence of the protection conferred by both endogenous and exogenous estradiol against colitis-associated tumorigenesis. Data provided in Table 1 summarizes the microscopic incidence of colonic neoplasms. Adenocarcinoma that developed in the 
Table 1. Incidence and multiplicity of adenoma and cancer in colon

\begin{tabular}{|c|c|c|c|c|c|c|}
\hline Group & $\begin{array}{l}\text { Low grade } \\
\text { adenoma } \\
\text { incidence }\end{array}$ & $\begin{array}{c}\text { High grade } \\
\text { adenoma } \\
\text { incidence }\end{array}$ & $\begin{array}{c}\text { Cancer with } \\
\text { mucosa } \\
\text { invasion }\end{array}$ & $\begin{array}{l}\text { Cancer with } \\
\text { submucosa } \\
\text { invasion }\end{array}$ & $\begin{array}{c}\text { Adenoma/ } \\
\text { cancer } \\
\text { incidence }\end{array}$ & $\begin{array}{c}\text { Adenoma/ } \\
\text { cancer } \\
\text { multiplicity }\end{array}$ \\
\hline \multicolumn{7}{|l|}{ 4-Week male } \\
\hline Control $(n=4)$ & $0 / 4(0)$ & $1 / 6(16.7)$ & $0 / 4(0)$ & $0 / 4(0)$ & $0 / 4(0)$ & 0.0 \\
\hline $\mathrm{AOM} / \mathrm{DSS}(\mathrm{n}=6)$ & $1 / 6(16.7)$ & $0 / 4(0)$ & $0 / 6(0)$ & $0 / 6(0)$ & $1 / 6(16.7)$ & $0.17 \pm 0.17$ \\
\hline $\mathrm{AOM} / \mathrm{DSS}+\mathrm{E} 2(\mathrm{n}=6)$ & $0 / 6(0)$ & $0 / 6(0)$ & $0 / 6(0)$ & $0 / 6(0)$ & $0 / 6(0)$ & $0.17 \pm 0.17$ \\
\hline$p$-value ${ }^{a)}$ & 1.000 & 1.000 & 1.000 & 1.000 & 1.000 & 0.762 \\
\hline p-value ${ }^{\text {b) }}$ & 1.000 & 1.000 & 1.000 & 1.000 & 0.455 & 1.000 \\
\hline $\mathrm{p}$-value ${ }^{\mathrm{c})}$ & 1.000 & 1.000 & 1.000 & 1.000 & 1.000 & 0.523 \\
\hline \multicolumn{7}{|l|}{ 4-Week female } \\
\hline Control $(n=4)$ & $0 / 4(0)$ & $0 / 4(0)$ & $0 / 4(0)$ & $0 / 4(0)$ & $0 / 4(0)$ & 0.0 \\
\hline AOM / DSS (n=6) & $0 / 6(0)$ & $1 / 6(16.7)$ & $0 / 6(0)$ & $0 / 6(0)$ & $1 / 6(16.7)$ & $0.33 \pm 0.21$ \\
\hline p-value ${ }^{a)}$ & 1.000 & 1.000 & 1.000 & 1.000 & 1.000 & 0.221 \\
\hline \multicolumn{7}{|l|}{ 10-Week male } \\
\hline Control $(n=6)$ & $0 / 6(0)$ & $0 / 6(0)$ & $0 / 6(0)$ & $0 / 6(0)$ & $0 / 6(0.0)$ & 0.0 \\
\hline AOM/DSS $(n=12)$ & $0 / 12(0)$ & $1 / 12(8.3)$ & $10 / 12(83.3)$ & $0 / 12(0)$ & $11 / 12(91.6)$ & $2.33 \pm 0.19$ \\
\hline $\mathrm{AOM} / \mathrm{DSS}+\mathrm{E} 2(\mathrm{n}=12)$ & $1 / 12(8.3)$ & $0 / 12(0)$ & $0 / 12(0)$ & $0 / 12(0)$ & $1 / 12(8.3)$ & 0.0 \\
\hline $\mathrm{p}$-value ${ }^{\mathrm{a})}$ & 1.000 & 1.000 & $0.002^{*}$ & 1.000 & $<0.001^{*}$ & $<0.001^{*}$ \\
\hline p-value ${ }^{\text {b) }}$ & 1.000 & 1.000 & $<0.001^{*}$ & 1.000 & $<0.001^{*}$ & $<0.001^{*}$ \\
\hline $\mathrm{p}$-value ${ }^{\mathrm{c}}$ & 1.000 & 1.000 & $0.012^{*}$ & 1.000 & 0.155 & $0.014^{*}$ \\
\hline \multicolumn{7}{|l|}{ 10-Week female } \\
\hline Control $(n=6)$ & $0 / 6(0)$ & $0 / 6(0)$ & $0 / 6(0)$ & $0 / 6(0)$ & $0 / 6(0)$ & 0.0 \\
\hline AOM/DSS $(n=12)$ & $1 / 12(8.3)$ & $2 / 12(16.6)$ & $3 / 12(25)$ & $1 / 12(8.3)$ & $7 / 12(58.3)$ & $1.17 \pm 0.39$ \\
\hline p-value ${ }^{a)}$ & 1.000 & 0.529 & 0.515 & 1.000 & $0.038^{*}$ & $0.025^{*}$ \\
\hline \multicolumn{7}{|l|}{ 16-Week male } \\
\hline Control $(n=6)$ & $0 / 6(0)$ & $0 / 6(0)$ & $0 / 6(0)$ & $0 / 6(0)$ & $0 / 6(0)$ & 0.0 \\
\hline AOM/DSS $(n=12)$ & $0 / 12(0)$ & $1 / 12(8.3)$ & $8 / 12(66.6)$ & $3 / 12(25)$ & $12 / 12(100)$ & $3.42 \pm 0.50$ \\
\hline $\mathrm{AOM} / \mathrm{DSS}+\mathrm{E} 2(\mathrm{n}=12)$ & $0 / 12(0)$ & $0 / 12(0)$ & $4 / 12(33.3)$ & $0 / 12(0)$ & 4/12 (33.3) & $1.83 \pm 0.34$ \\
\hline p-value ${ }^{a)}$ & 1.000 & 1.000 & $0.013^{*}$ & 0.515 & $<0.001^{*}$ & $0.001^{*}$ \\
\hline$p$-value ${ }^{b)}$ & 1.000 & 1.000 & 0.220 & 0.217 & $0.001^{*}$ & $0.020^{*}$ \\
\hline p-value ${ }^{c)}$ & 1.000 & 1.000 & 0.100 & 0.590 & $0.001^{*}$ & 0.243 \\
\hline \multicolumn{7}{|l|}{ 16-Week female } \\
\hline Control $(n=6)$ & $0 / 6(0)$ & $0 / 6(0)$ & $0 / 6(0)$ & $0 / 6(0)$ & $0 / 6(0)$ & 0.0 \\
\hline AOM/DSS $(n=12)$ & $0 / 12(0)$ & $0 / 12(0)$ & $3 / 12(25.0)$ & $1 / 12(8.3)$ & $4 / 12(33.3)$ & $2.42 \pm 0.71$ \\
\hline p-value ${ }^{a)}$ & 1.000 & 1.000 & 0.526 & 1.000 & 0.245 & $0.035^{*}$ \\
\hline
\end{tabular}

Values are expressed as number/subtotal (\%) or mean \pm SEM. AOM, azoxymethane; DSS, dextran sulphate sodium; E2, 17 $\beta$ -

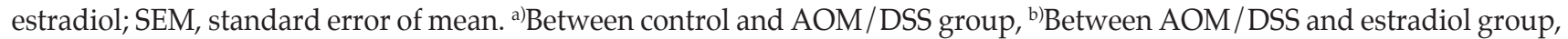
${ }^{c}$ Between male and female. Fisher exact test, ${ }^{*} \mathrm{p}<0.05$.

M-AOM/DSS group tended to have invasive growth, while that in the M-AOM/DSS+estr group displayed an intact muscularis mucosa lining at week 10 (Fig. 2C). The M-AOM/ DSS group presented the most severe histological invasiveness at week 16 (Fig. 2D).

\section{Effect of estradiol during colitis and cancer progression in terms of NF- $\mathrm{KB}$}

To further evaluate the estradiol effects on inflammatory factors at the molecular level, we measured NF- $\mathrm{kB}$ and its related pro-inflammatory enzymes, cytokines, and genes. First of all, we determined the expression levels of NF- $\mathrm{B}$ by Western blot analysis at week 2 . The M-AOM/DSS group had higher levels of NF- $\mathrm{B}$, compared to both the F-AOM/ 

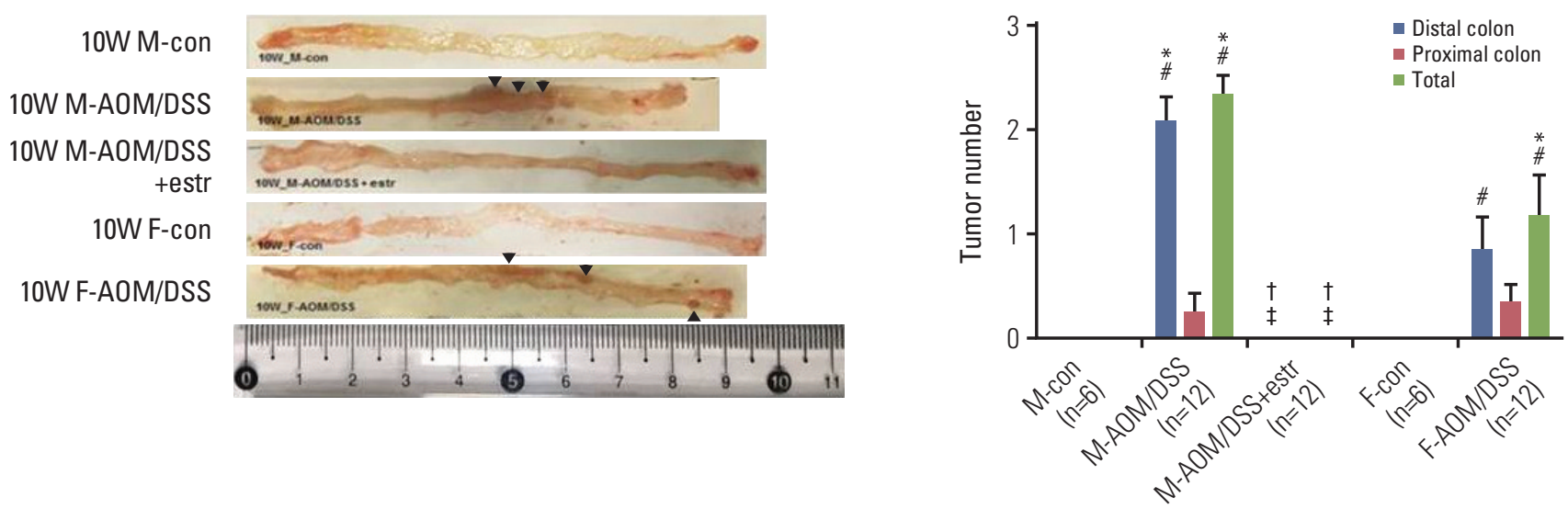

16W M-con

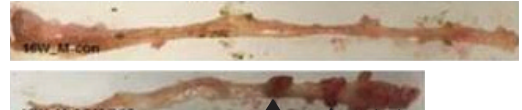

16W M-AOM/DSS

16W M-AOM/DSS

+estr

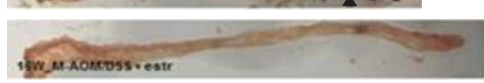

16W F-con

16W F-AOM/DSS
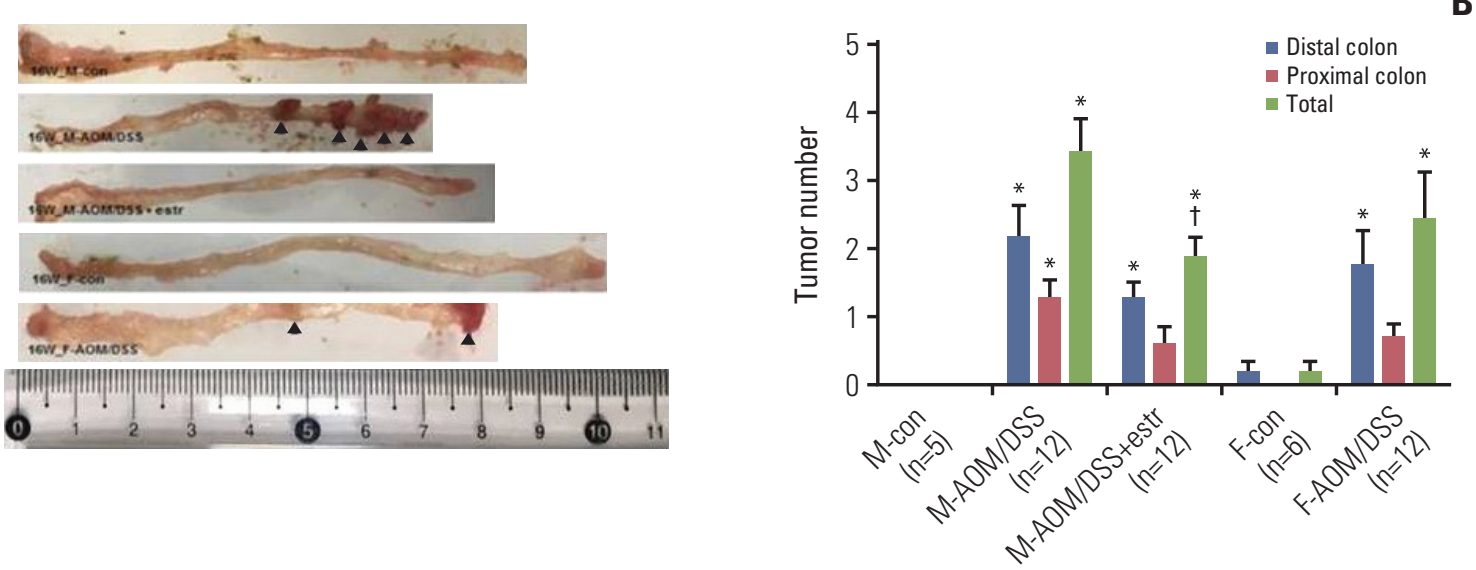

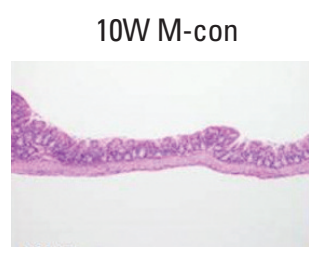

10W F-con
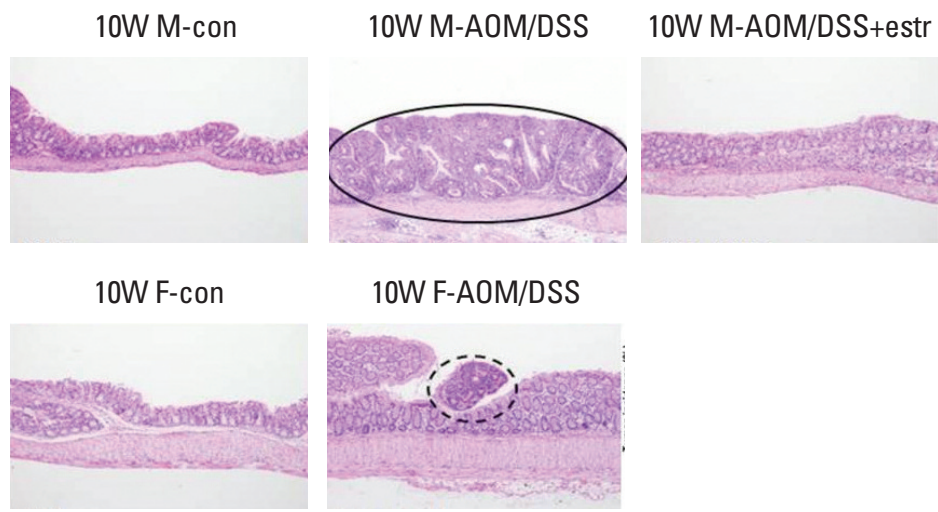

\begin{abstract}
10W F-AOM/DSS
\end{abstract}

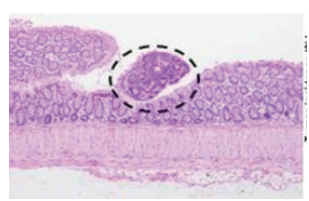

B

C

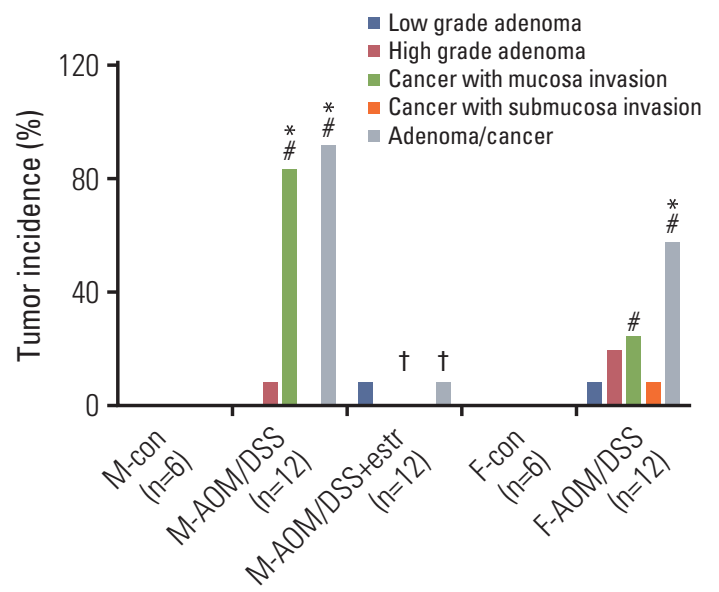

Fig. 2. Effect of estradiol and sex-associated differences in the multiplicity of colorectal cancer at weeks 10 and 16 . Macroscopic view (left panel) and multiplicity of the colons (right panel) in each group sacrificed at weeks 10 (A) and 16 (B). Arrowheads indicate the macroscopic polyps. Representative histological images at weeks 10 (C) and 16 (D). (Continued to the next page) 
16W M-con

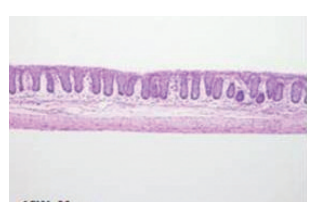

16W F-con

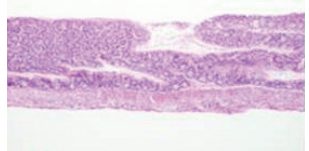

16W M-AOM/DSS

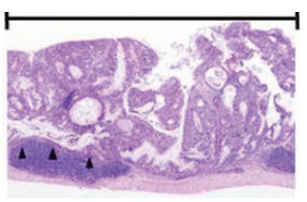

16W F-AOM/DSS

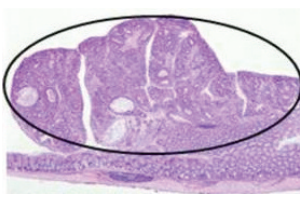

16W M-AOM/DSS+estr

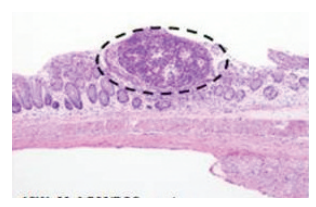

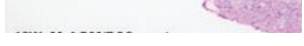

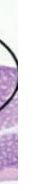

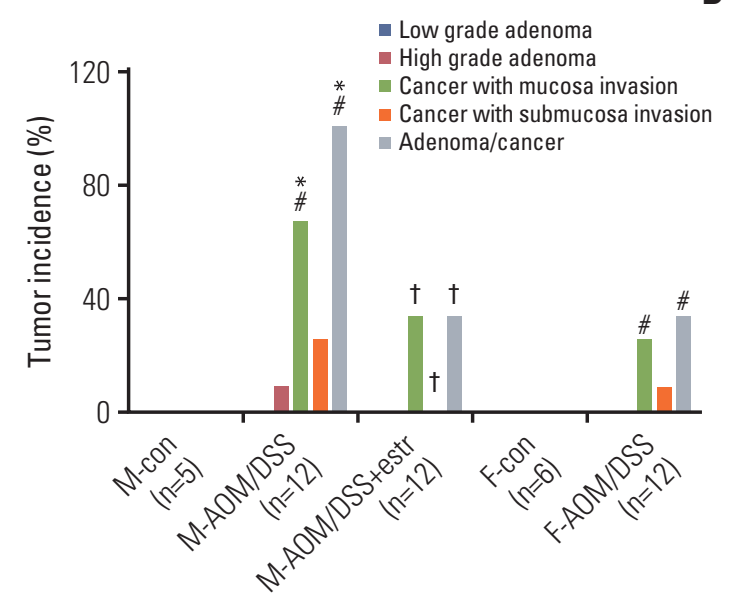

Fig. 2. (Continued from the previous page) Adenoma is indicated with dashed line circle, adenocarcinoma with full line circle and a bar, and submucosal invasion with arrowheads. Quantification of invasion and incidence of cancer in each group at 10 and 16 weeks obtained by microscopic evaluation of the colonic tissues (H\&E staining, $\times 100$ ). ${ }^{*} p<0.05$ compared to control, ${ }^{\dagger} \mathrm{p}<0.05$ compared to the in azoxymethane/ dextran sulfate sodium (AOM/DSS) group, ${ }^{\ddagger} \mathrm{p}<0.05$ between the estradiol-treated group and the female AOM/DSS group, ${ }^{*} \mathrm{p}<0.05$ between the male AOM/DSS group and the female AOM/DSS group. M, male; F, female; estr, estradiol.

DSS and M-AOM/DSS+estr groups (Fig. 3A). Consistent with the NF-kB results, the levels of two major pro-inflammatory enzymes (cyclooxygenase-2 [COX-2] and inducible nitric oxide synthase [iNOS]), which are mainly regulated by $\mathrm{NF}-\mathrm{kB}$, were higher in the M-AOM/DSS group, compared to the F-AOM/DSS group ( $\mathrm{p}<0.05$ for iNOS) (Fig. 3A). The protein and mRNA levels of NF-kB-related pro-inflammatory enzymes were decreased in the M-AOM/DSS+estr group compared to the M-AOM/DSS group at week 2, to similar levels of control mice $(\mathrm{p}<0.05$ for COX-2) (Fig. 3A and B). Next, we measured NF-kB-related pro-inflammatory cytokines (i.e. interleukin 6 [IL-6] and tumor necrosis factor $\alpha[$ TNF- $\alpha]$ ) in the colonic mucosa at week 2 by real-time quantitative reverse transcription PCR (qRT-PCR). The F-AOM/ DSS group displayed reduced levels of the pro-inflammatory cytokines compared to the M-AOM/DSS group at week 2 (Fig. 3B). Moreover, decreased levels of IL-6 and TNF- $\alpha$ were observed in the M-AOM/DSS+estr group compared to the M-AOM/DSS group (both $p<0.05$ ) (Fig. 3B). The proinflammatory enzyme levels and cytokine gene expression at week 2 were consistent with the results of DAI and microscopic damage index at week 2 . These data suggest that NF- $\mathrm{kB}$ and NF- $\mathrm{kB}-$ related pro-inflammatory mediators are the molecular basis of the sex difference in colitis. These observations also suggest that estradiol exerts anti-inflammatory effects by suppressing NF-kB-related pro-inflamma- tory mediators. NF- $\kappa \mathrm{B}-$ related pro-inflammatory mediators were consistently expressed at high levels in the M-AOM/ DSS-group (Fig. 3C-F).

\section{Effect of estradiol during colitis and cancer progression in terms of Nrf2}

Since Nrf2 directly downregulates NF-kB expression and activity [13], we next investigated $\mathrm{Nrf2}$ and its related antioxidant enzyme activation.

The IHC analysis of Nrf2 showed significant increase of Nrf2 by estradiol at week $2(p<0.05)$ (Fig. $4 \mathrm{~A}$ and $\mathrm{B})$, and by AOM/DSS at weeks 10 and 16 (p $<0.05$ at week $10, p<0.01$ at week 16) (Fig. 4E, F, I, and J). The proportion of Nrf2immunostained cells in crypts was significantly higher in females than in males on weeks 10 and 16 (all $\mathrm{p}<0.05$ ) (Fig. 4E, F, I, and J). At week 16, the Nrf2-immunostained cells were decreased by estradiol $(\mathrm{p}<0.05)$.

The F-AOM/DSS group showed higher expression of Nrf2 compared to the M-AOM/DSS group in terms of the levels of protein (Fig. 4C) and mRNA (Fig. 4D) at week 2. Nuclear translocation and mRNA expression of Nrf2 were increased in the M-AOM/DSS+estr group compared to the M-con and M-AOM/DSS groups at week 2 ( $<<0.001$ ) (Fig. 4C and D). The protein and mRNA levels of $\mathrm{PKC} \delta$, which positively regulate Nrf2 [8,21], also increased in the M-AOM/DSS+estr 
A
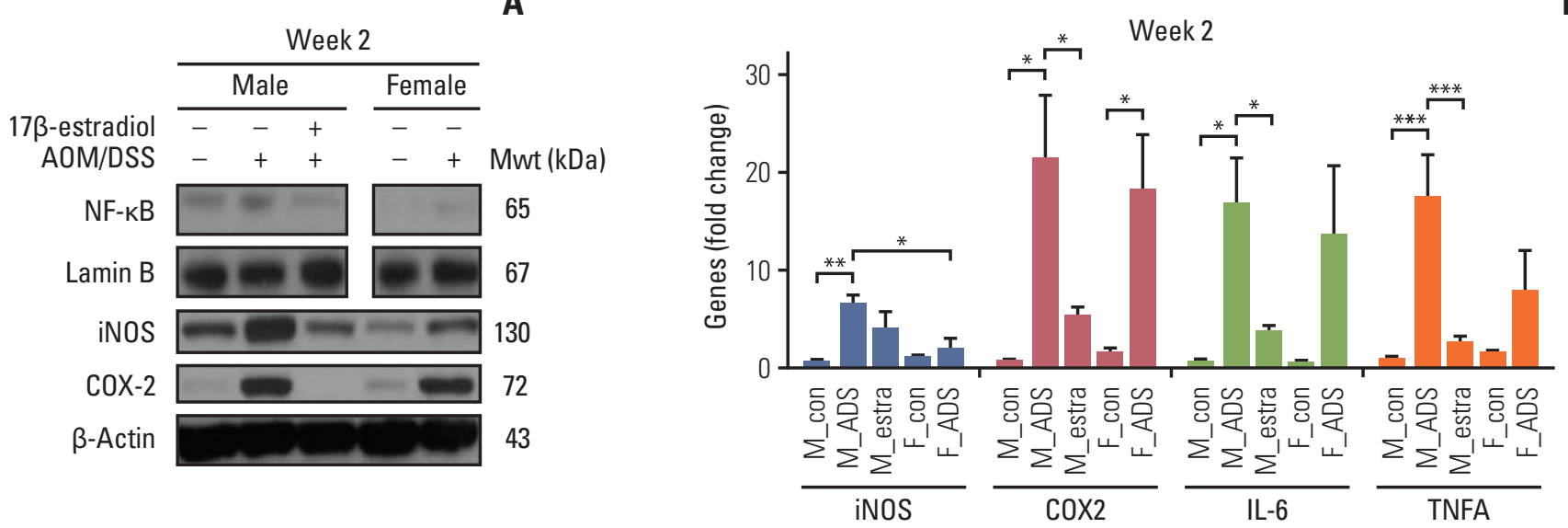

C
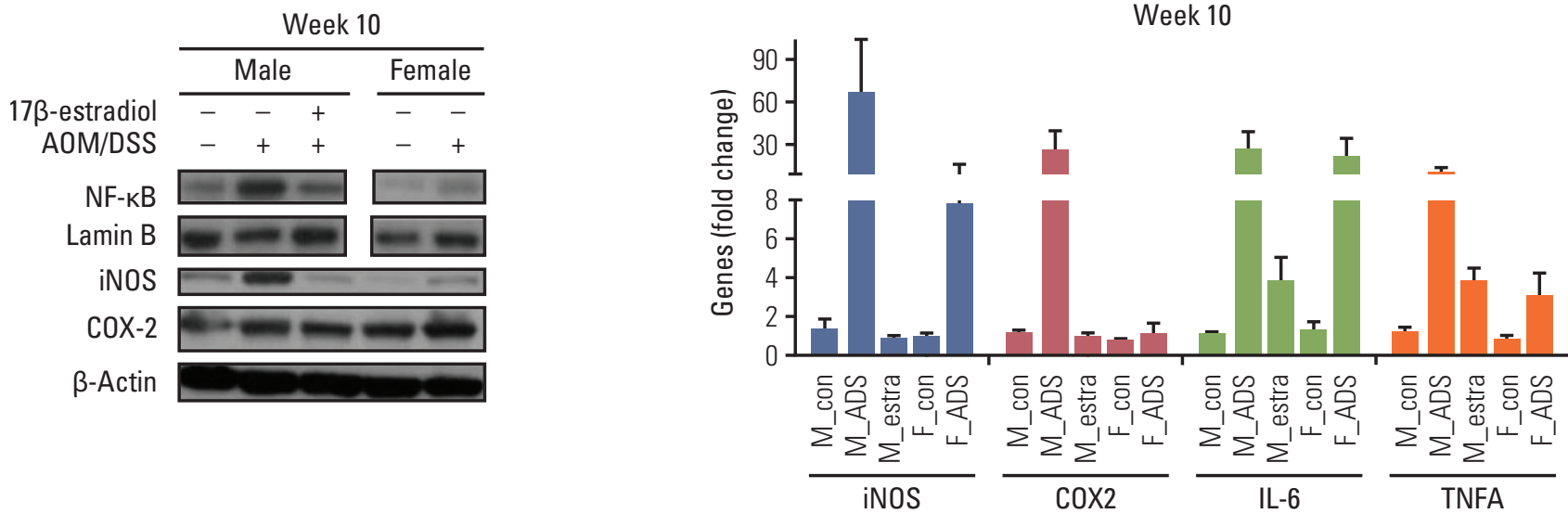

E
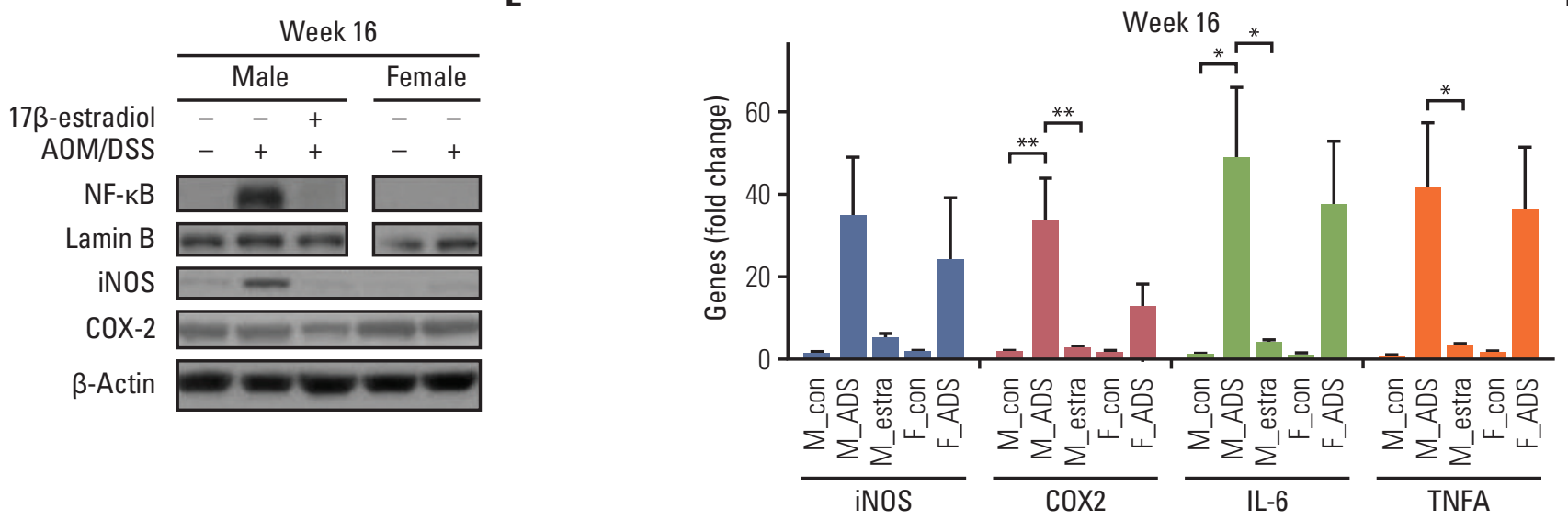

Fig. 3. Protein and $m R N A$ expression levels of nuclear factor $\kappa B(N F-\kappa B)$ and its related pro-inflammatory factors in colonic tissues at weeks $2(\mathrm{~A}, \mathrm{~B}), 10(\mathrm{C}, \mathrm{D})$, and $16(\mathrm{E}, \mathrm{F})$. Western blot analysis of NF-kB, inducible nitric oxide synthase (iNOS), and cyclooxygenase 2 (COX2) at weeks 2 (A), 10 (C), and 16 (E). mRNA expression levels of iNOS, COX2, interleukin 6 (IL-6), and TNFA, determined with real-time polymerase chain reaction, at weeks $2(B), 10(D)$, and $16(\mathrm{~F}) .{ }^{*} \mathrm{p}<0.05,{ }^{* *} \mathrm{p}<0.01$, and ${ }^{* * *} \mathrm{p}<0.001$. M, male; F, female; AOM, azoxymethane; DSS, dextran sulfate sodium; estra, estradiol. 

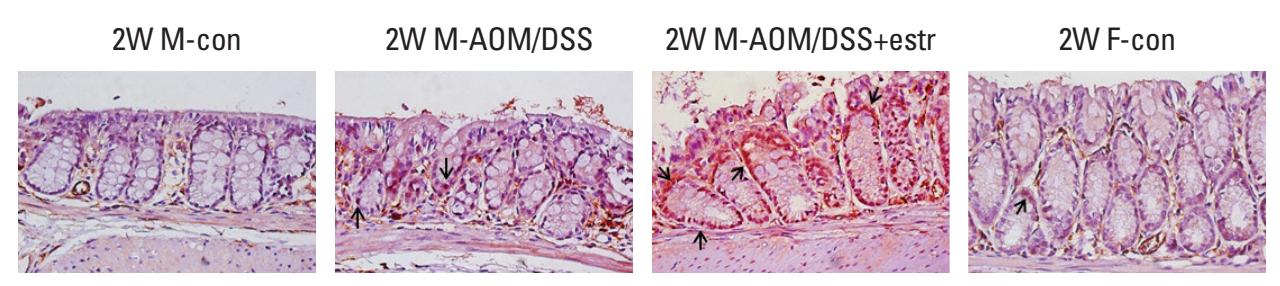

\section{W F-AOM/DSS}

A

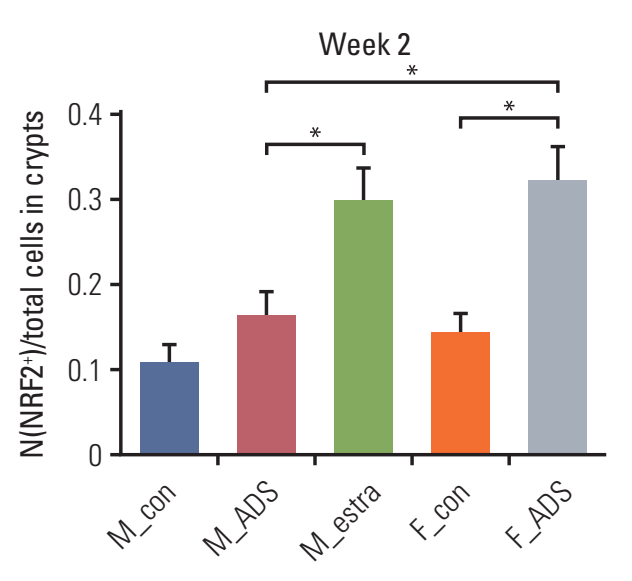

B

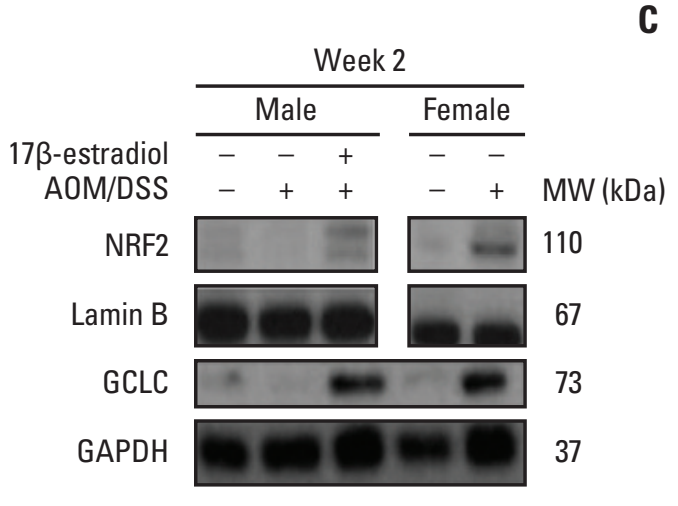

C

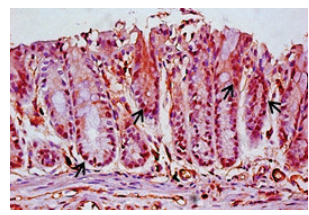

Week 2

D

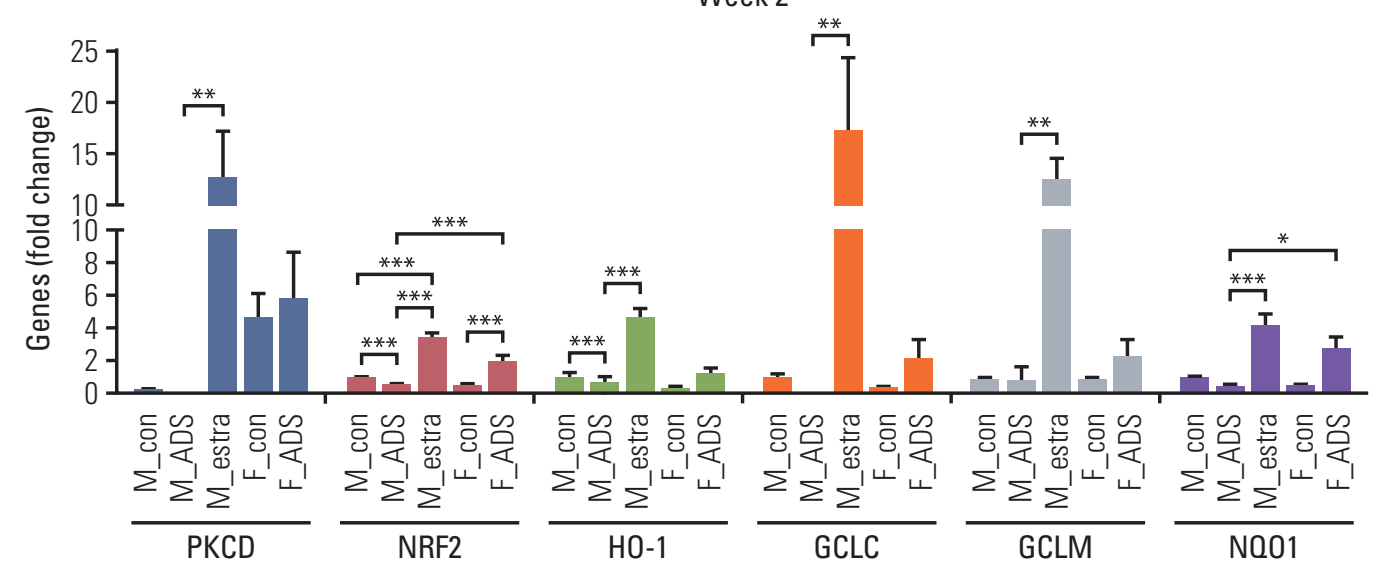

Fig. 4. Expression levels of nuclear factor erythroid 2-related factor 2 (NRF2) and its related anti-oxidant enzymes in colonic tissues at weeks 2 (A-D), 10 (E-H), and 16 (I-L). Photomicrography of NRF2 immunostain of distal mouse colon at weeks 2 (A), $10(\mathrm{E})$, and 16 (I). Arrows indicate the NRF2-immunoreactive cells $(\times 400)$. Analysis of NRF2 immunohistochemistry in distal colonic tissues at week 2 (B), 10 (F), and 16 (J). Western blot analysis of NRF2 and glutamate-cysteine ligase catalytic subunit (GCLC) at weeks $2(\mathrm{C}), 10(\mathrm{G})$, and $16(\mathrm{~K})$. mRNA expression levels of PKCD, NRF2, HO-1, GCLC, GCLM, and NQO-1, determined with real-time polymerase chain reaction, at weeks 2 (D), $10(\mathrm{H})$, and $16(\mathrm{~L}) .{ }^{*} \mathrm{p}<0.05$, ** $\mathrm{p}<0.01$ and ${ }^{* * *} \mathrm{p}<0.001$. M, male; F, female; AOM, azoxymethane; DSS, dextran sulfate sodium; ADS, AOM/DSS; estra, estradiol; MW, molecular weight; GAPDH, glyceraldehyde 3-phosphate dehydrogenase. (Continued to the next page) 
10W M-con

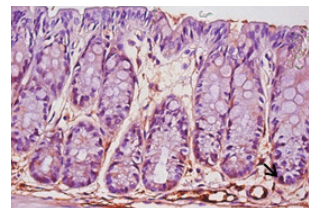

10W M-AOM/DSS

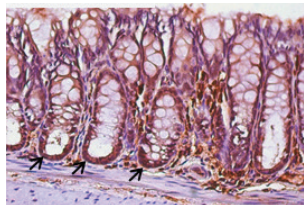

10W M-AOM/DSS+estr

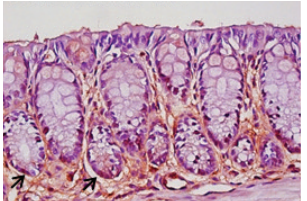

10W F-con

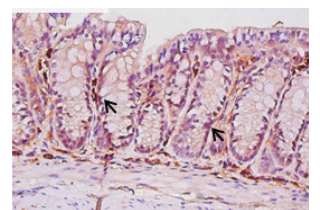

10W F-AOM/DSS

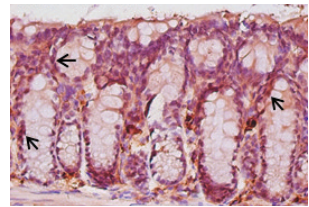

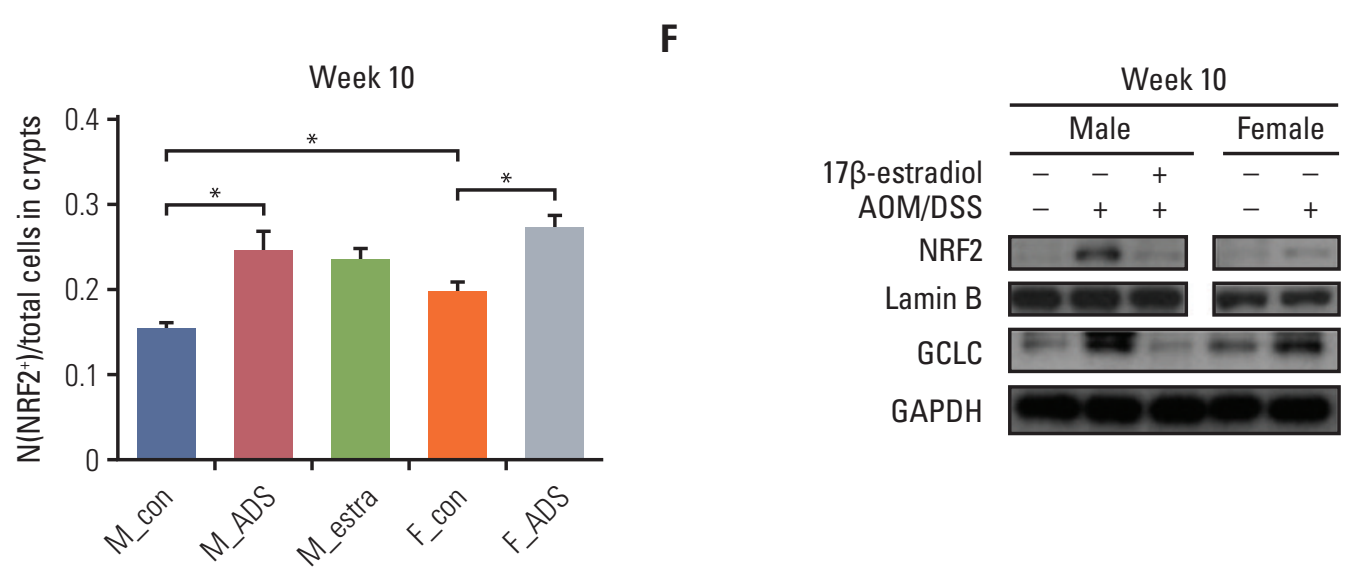

G

Week 10

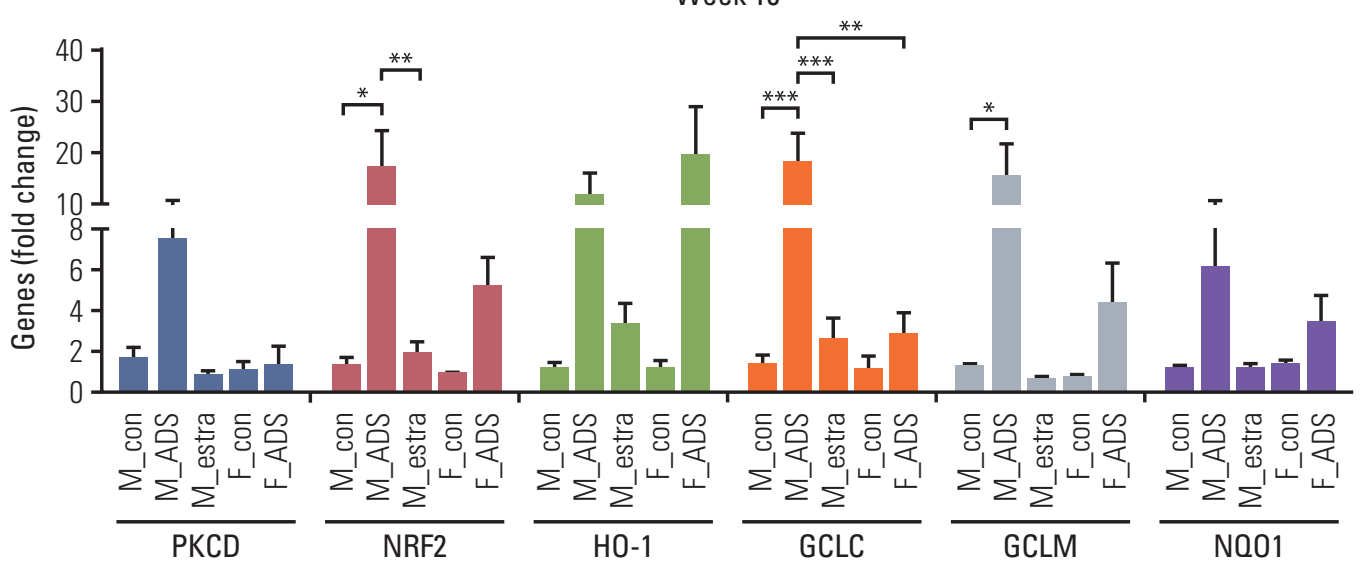

Fig. 4. (Continued from the previous page) (Continued to the next page)

group compared to the M-AOM/DSS group (all $p<0.05$ ) (Fig. 4D and S3 Fig. of the SI). The F-AOM/DSS and F-con groups also showed higher mRNA levels of PKC $\delta$ compared to the M-AOM/DSS and M-con groups (all p < 0.05) (Fig. 4D). In addition, $\mathrm{G} \alpha_{13}$ strengthens ER $\alpha$ activity [22], and estradiol is closely related to the activities of PKC $\delta$ [11] and Nrf2 [7]. Another G protein, $G \alpha_{12}$, regulates NF- $\mathrm{KB}$ in an estradiol-independent pathway [23]. When we measured the $\mathrm{G}_{13}$ and $\mathrm{G} \alpha_{12}$ by Western blot analysis, only $\mathrm{G} \alpha_{13}$ was signif- icantly increased in the M-AOM/DSS+estr group compared to the M-AOM/DSS group ( $p<0.001$ ) (S3 Fig.). G $\alpha_{13}$ expression of the F-AOM/DSS group was significantly higher than the M-AOM/DSS ( $p$ < 0.001) (S3 Fig.). Taken together, these data strongly suggest that activation of Nrf2 by exogenous and endogenous estradiol is closely correlated to the activation of $\mathrm{G} \alpha_{13}-\mathrm{PKC} \delta$ signaling pathway during DSS-induced inflammation stage at week 2.

Nrf2 activation also resulted in up-regulation of anti-oxi- 


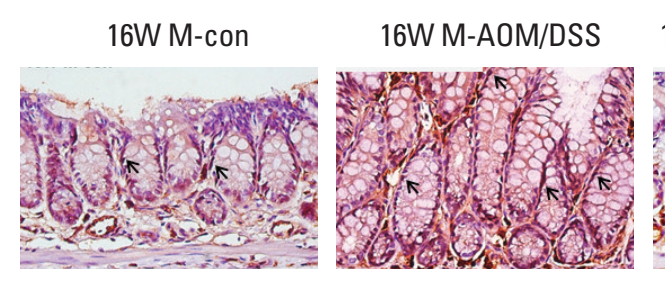

16W M-AOM/DSS+estr

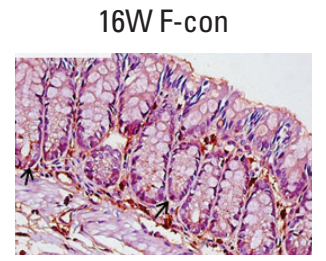

16W F-AOM/DSS
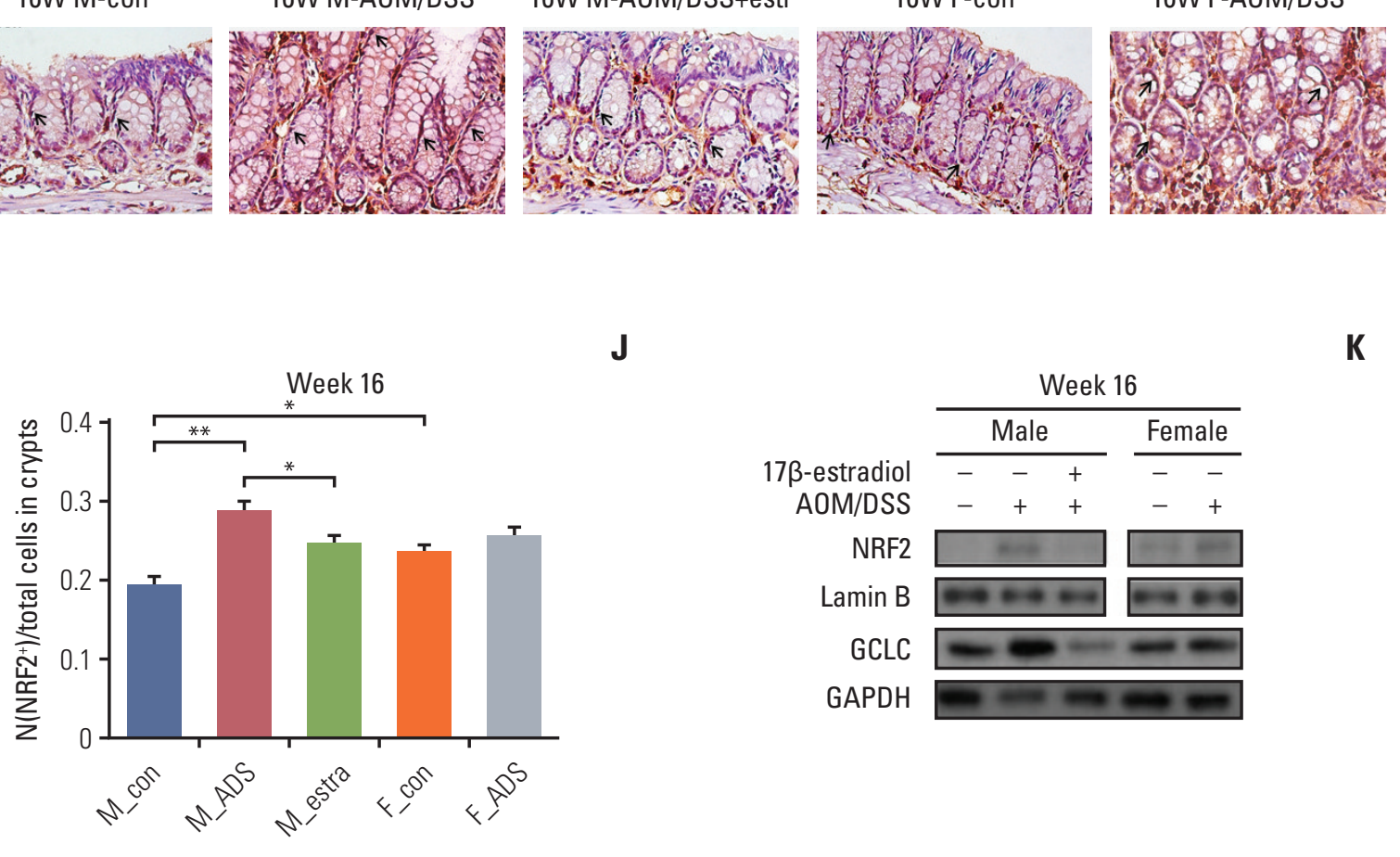

K

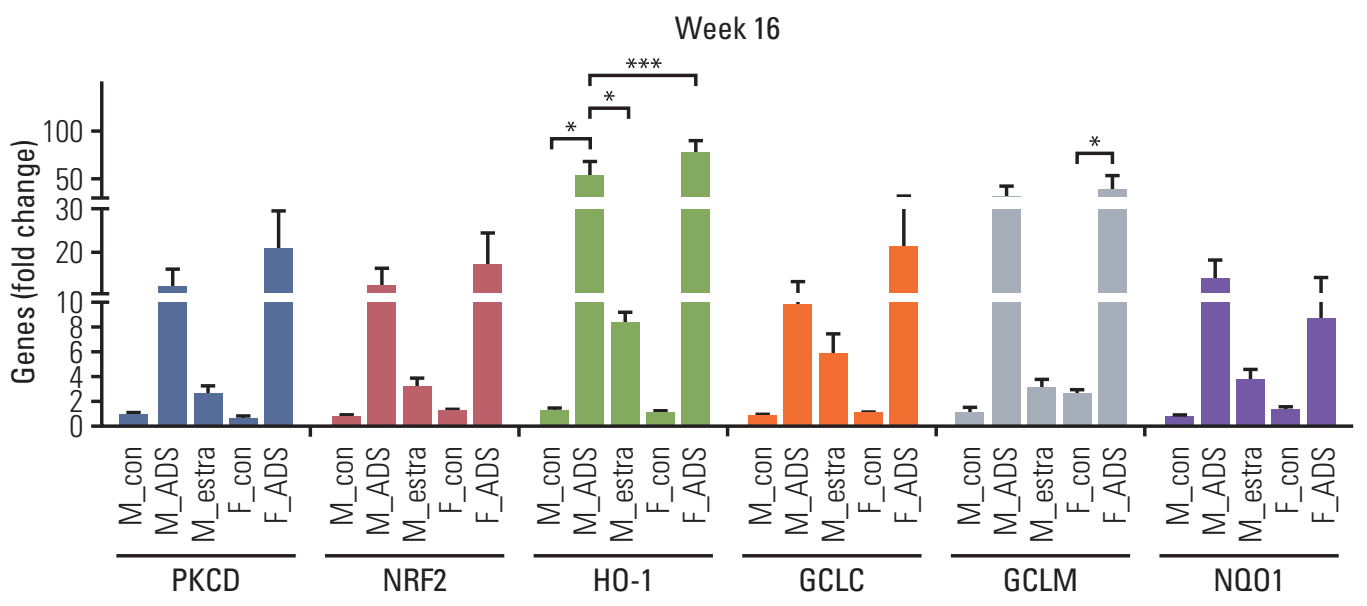

Fig. 4. (Continued from the previous page)

dant gene expression at week 2. Glutamate-cysteine ligase catalytic subunit (GCLC) is an antioxidant enzyme regulated by Nrf2. GCLC was increased in the F-AOM/DSS and $\mathrm{M}-\mathrm{AOM} / \mathrm{DSS}+\mathrm{estr}$ groups compared to control mice and the M-AOM/DSS group at week 2 (Fig. 4C). This observation is consistent with the increased mRNA expression of other antioxidant enzymes (i.e., HO-1, GCLM, and NQO-1) in the F-AOM/DSS and M-AOM/DSS+estr groups compared to control mice and the M-AOM/DSS group at week 2 (all $\mathrm{p}<0.05$ for M-AOM/DSS+estr group vs. M-AOM/DSS group, $\mathrm{p}<0.001$ for NQO-1 between F-AOM/DSS group and M-AOM/DSS group) (Fig. 4D). These data suggest that both endogenous and exogenous estradiol relieve DSS-induced colitis by promoting anti-oxidant gene expression through Nrf2 activation.

We investigated Nrf2 expression levels by Western blot analysis at weeks 10 and 16 . At these times, CRC had developed in the AOM/DSS model. In contrast to week 2, both 
A

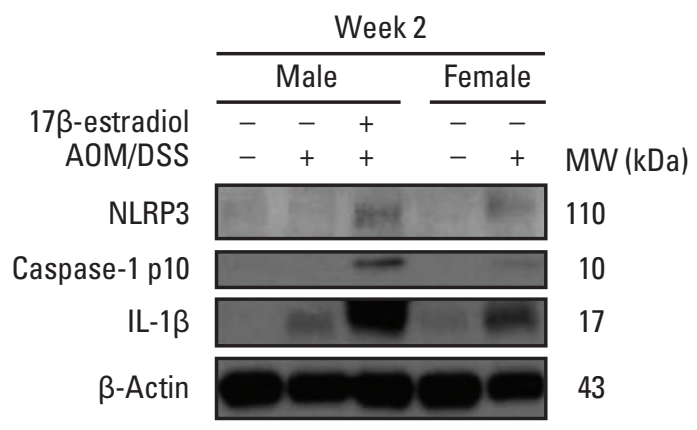

C

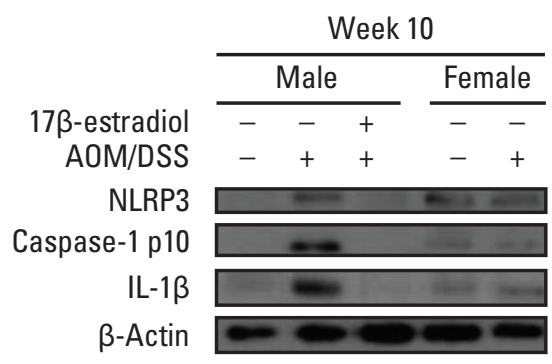

$\mathbf{E}$

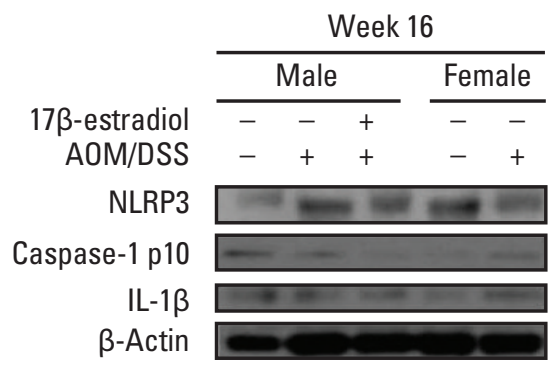

Week 2

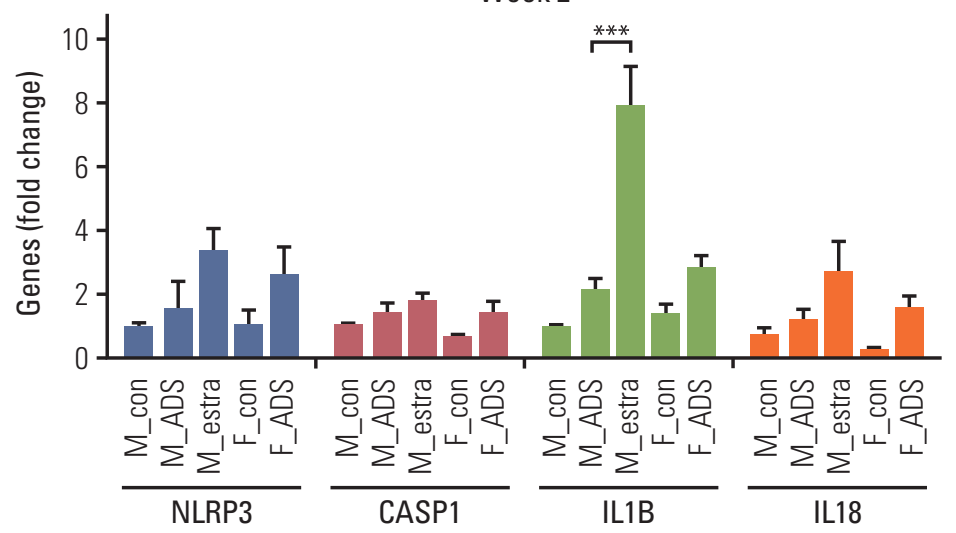

D

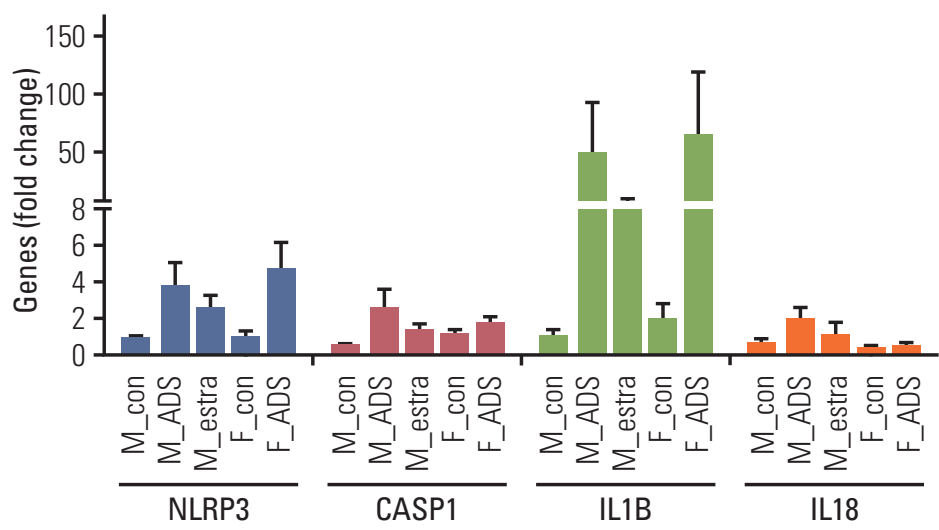

$\mathbf{F}$

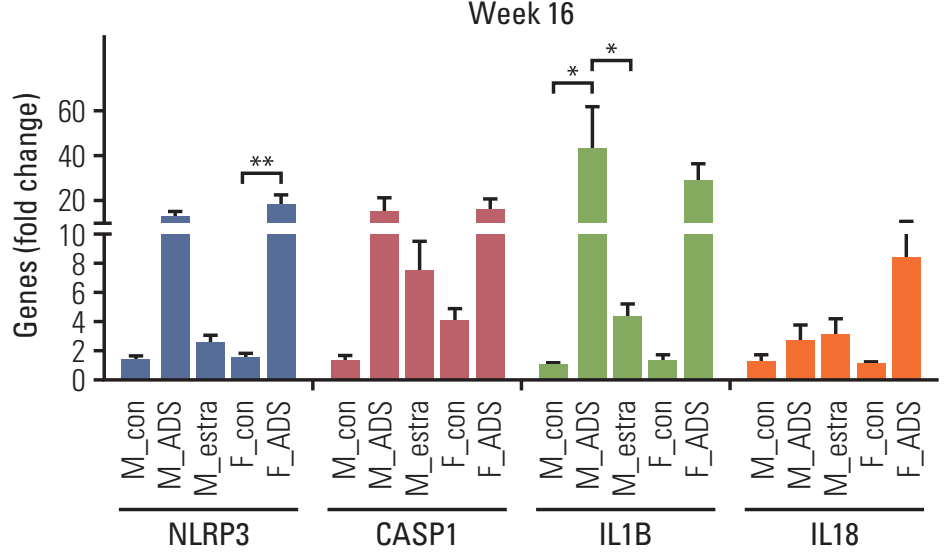

Fig. 5. Protein and mRNA level analyses of Nod-like receptor protein 3 (NLRP3) inflammasome activation in colonic tissues at weeks $2(\mathrm{~A}, \mathrm{~B}), 10(\mathrm{C}, \mathrm{D})$, and $16(\mathrm{E}, \mathrm{F})$. Western blot analysis of NLRP3, caspase-1 p10, and interleukin (IL)-1 $\beta$ at weeks 2 (A), 10 (C), and 16 (E). mRNA expression levels of NLRP3, CASP1, IL1B, and IL18, determined with real-time polymerase chain reaction, at weeks 2 (B), 10 (D), and 16 (F). ${ }^{*} \mathrm{p}<0.05,{ }^{* *} \mathrm{p}<0.01$, and ${ }^{* * *} \mathrm{p}<0.001$. M, male; F, female; AOM, azoxymethane; DSS, dextran sulfate sodium; MW, molecular weight; ADS, AOM/DSS; estra, estradiol. 
A
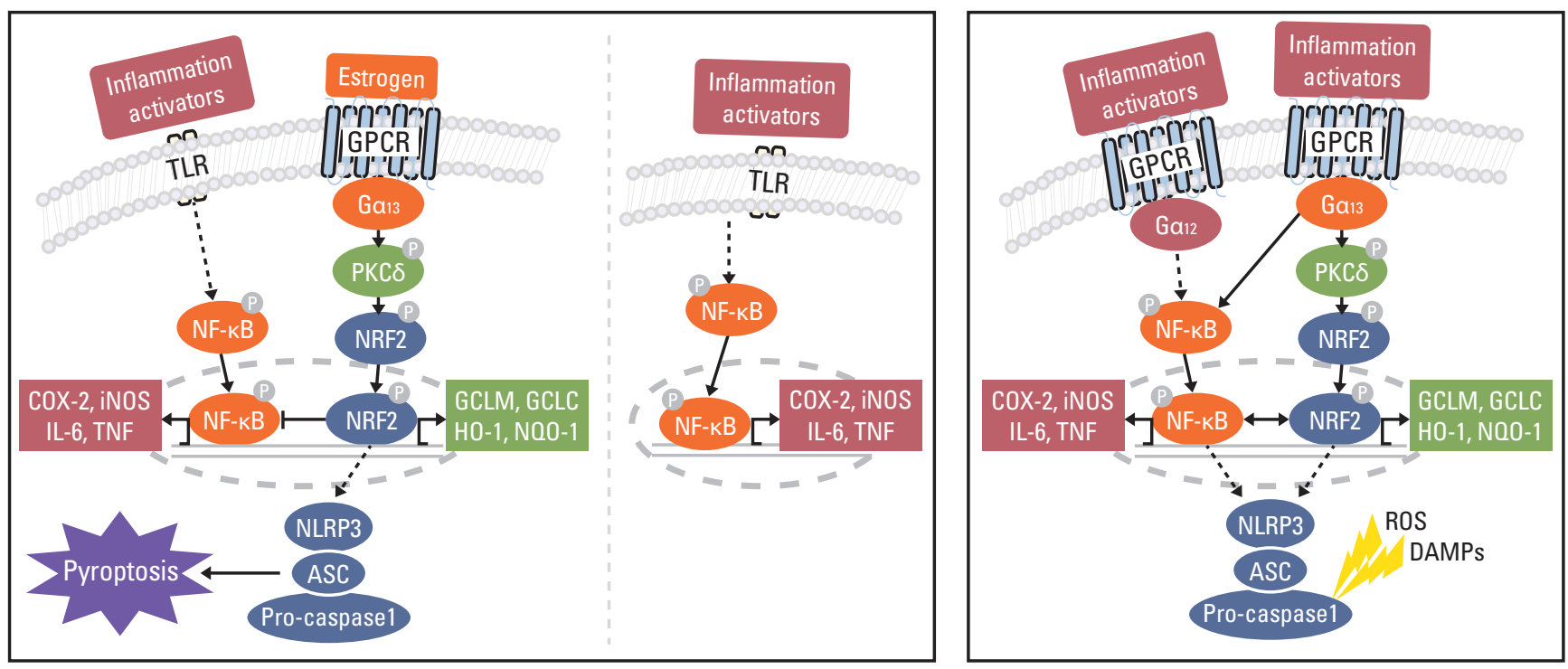

Multistep activation

Stimulates

Inhibits

$\rightarrow$ Interaction

Fig. 6. Proposed regulatory mechanism of estrogen in colitis-associated colorectal cancer at week 2 (A) and at weeks 10 and 16 (B). (A) Estrogen induces inflammasome activation through G $\alpha_{13}$ protein subunits. G $\alpha_{12}$ and G $\alpha_{13}$ have potentiated estrogen-bound estrogen receptor $\alpha$ activity. However, despite the functional overlap between $\mathrm{G} \alpha_{12}$ and $\mathrm{G} \alpha_{13}$, only $\mathrm{G} \alpha_{13}$ regulates nuclear factor erythroid 2-related factor 2 (Nrf2) via protein kinase $\mathrm{C} \delta(\mathrm{PKC} \delta)$. Nrf2 mediates inflammasome activation through the transcription of as-yet unknown genes. Nod-like receptor protein 3 (NLRP3) inflammasome activation induces pyroptosis to eliminate precancerous cells. After eliminating precancerous cells, Nrf2 inhibits nuclear factor $\kappa \mathrm{B}$ (NF- $\kappa \mathrm{B})$ and reactive oxygen species through the anti-oxidant enzymes. Ultimately, estrogen prevents carcinogenesis (left panel). In contrast, in the absence of estrogen, inflammation provides a cancer microenvironment through activation of the NF- $\mathrm{kB}$ pathway (right panel). (B) After unsuccessful elimination of precancerous cells, inflammation progresses to cancer at weeks 10 and 16. G $\alpha_{12}$ and $\mathrm{G} \alpha_{13}$ regulate $\mathrm{NF}-\kappa \mathrm{B}$ and $\mathrm{Nrf} 2$ via PKC $\delta$-mediated signaling pathway, respectively. Nrf2 promotes tumor progression by activation of anti-oxidant enzymes and NLRP3 inflammasome. Ultimately, NF- $\mathrm{B}$ and Nrf2 signaling pathway accelerate carcinogenesis. COX-2, cyclooxygenase 2; DAMP, damage-associated molecular pattern; GPCR, G protein coupled receptor; IL, interleukin; iNOS inducible nitric oxide synthase; ROS, reactive oxygen species; TLR, Toll-like receptor; TNF, tumor necrosis factor.

protein and mRNA levels of Nrf2 were higher in the M-AOM/DSS group compared to the M-AOM/DSS+estr group at weeks 10 and 16 ( $\mathrm{p}<0.01$ at week 10) (Fig. 4E-L). Consistent with Nrf2, GCLC and other anti-oxidant enzymes were highly expressed in the M-AOM/DSS-group at weeks 10 and 16 (Fig. 4H and L). These findings suggest that, after tumor initiation, Nrf2 expression might overly induce antioxidant enzymes that could somehow result in a tumor microenvironment that was favorable to tumor progression. This explanation was supported by the cancer developmental factor analysis at the F-AOM/DSS group. That is, $25 \%$ of weeks 10 and 16 (both $n=12$ ) showed CRC development; they were divided into the cancer group (both $n=4$ ) and non-cancer group (both $n=8$ ) (S4 Fig.). The F-AOM/DSS cancer group showed significantly higher levels of $\mathrm{HO}-1$ and PKC $\delta$ than the F-AOM/DSS non-cancer group at week 16 ( $\mathrm{p}<0.05$ for PKC $\delta$ ) (S4 Fig.). Other factors, such as NQO1, GCLM, iNOS, and Nrf2, were elevated in the F-AOM/DSS cancer group compared to the F-AOM/DSS non-cancer group, but did not reach statistical significance (S4 Fig.).

\section{Effect of estradiol during colitis and cancer progression in terms of NLRP3}

The close relationship of Nrf2 with the activating mechanism of NLRP3 inflammasome, which finally activates IL-1 $\beta$ and IL-18 [16], inspired the present measurement of protein and mRNA levels of the NLRP3 inflammasome and its 
related mediators by Western blot (Fig. 5A) and qRT-PCR analyses (Fig. 5B), to investigate its relationship with Nrf2 in AOM/DSS mice. Consistent with the Nrf2-mediated activation and up-regulation of anti-oxidant gene expression at week 2, the Western blot analysis clearly showed an increase of the NLRP3 inflammasome and its related phosphorylated caspase- 1 and IL- $1 \beta$ in the F-AOM/DSS and M-AOM/DSS+ estr groups compared to the M-AOM/DSS group at week 2 ( $p<0.05$ for NLRP3 and IL-1 $\beta$ between the M-AOM/DSS+ estr and M-AOM/DSS groups) (Fig. 5A, S3 Fig.). The data for mRNA expression definitely supported the significant increase of IL-1 $\beta$ in the M-AOM/DSS+estr group compared to the M-AOM/DSS group, $(\mathrm{p}<0.001)$ (Fig. $5 \mathrm{~B})$.

Next, we examined the expression of NLRP3 inflammasome-related enzymes and mediators (NLRP3, caspase 1, IL-1 $\beta$, and IL-18) at weeks 10 and 16 by Western blot analysis. Notably, the expressions of caspase- 1 and IL-1 $\beta$ were elevated in the M-AOM/DSS group compared to the M-AOM/ DSS+estr group at weeks 10 and 16 in both protein (Fig. 5C and E) and mRNA analyses (Fig. 5D and F). Similarly, the F-AOM/DSS cancer group also showed higher mRNA levels of NLRP3 inflammasome-related enzymes and mediators compared to the F-AOM/DSS non-cancer group ( $p<0.05$ for NLRP3 and caspase- 1 at week $10, p<0.05$ for NLRP3 at week 16) (S4 Fig.). These data suggest that once a tumor is initiated, the NLRP3 inflammasome might promote tumor development, which is consistent with the Nrf2 data.

\section{Discussion}

After confirming the sex difference in CRC development by showing that the F-AOM/DSS group has significantly lower tumor multiplicity and incidence compared with the $\mathrm{M}-\mathrm{AOM} / \mathrm{DSS}$ group, we further investigated the underlying anti-cancer mechanism of estradiol. Our findings demonstrate a dual role of Nrf2 in modulating inflammation and carcinogenesis through the regulation of the NF- $\mathrm{kB}$-mediated pro-inflammatory pathway, anti-oxidant enzymes, and NLRP3 inflammasome. In this research, we focused on week 2, which is the active DSS-induced inflammation stage [20], just after the completion of estradiol administration, and several weeks before the AOM/DSS-induced tumorigenesis. The severity of inflammation at week 2 was associated with tumor formation at weeks 10 and 16. Our study clearly demonstrates the importance of early inflammatory control by the administration of estradiol to AOM/DSS-treated male mice, to confirm the role of estradiol for CRC prevention. In addition, the M-AOM/DSS+estr group was compared with the F-AOM/DSS group to check any differences between exogenous and endogenous estradiol. This approach strongly supports the inhibitory effect of estradiol on inflammation and inflammation-induced tumorigenesis, and also notably uncovers its underlying mechanism of estradiol in three aspects: (1) NF-kB-related pro-inflammatory mediators, (2) Nrf2-related anti-oxidant enzymes, and (3) NLRP3 inflammasome.

The NF-kB signaling pathway is highly involved in inflammation and cancer development, especially in colitis-associated CRC [24]. In the present study, estradiol inhibited the NF- $\mathrm{KB}$ signaling pathway involving iNOS, COX-2, IL-6, and TNF- $\alpha$ during the DSS-induced inflammation stage of AOM/ DSS-induced colon tumorigenesis. Furthermore, the concomitant increased expression of $\mathrm{G}_{13}$, PKC $\delta$, and Nrf2 by estradiol administration in AOM/DSS-treated male mice during the DSS-induced inflammation stage supports the correlation between the suppression of NF- $\mathrm{kB}$ signaling and the estradiol-induced activation of $\mathrm{G}_{13} / \mathrm{PKC} \delta / \mathrm{Nrf} 2$ pathway. ERs inhibit the NF-kB pathway by the modulation of upstream signaling or the transcriptional activation of $\mathrm{NF}-\kappa \mathrm{B}$ in various cell lines [25]. Nrf2 inhibition of NF- $\kappa \mathrm{B}$ activity is well known. Ga13 strengthens ER $\alpha$ activity [22], and estradiol regulates the activity of PKC $\delta$ [11], and Nrf2 [7]. To further support the estradiol-induced activation of Nrf2 pathway, we are performing experiments using Nrf2 Knockout mice.

It has been reported that anti-oxidant enzymes activated by Nrf2 have cancer preventive effects by eliminating reactive oxygen species, and facilitating the resolution of inflammation [26]. In the present study, the levels of Nrf2 and NQO-1 expression were different between the M-AOM/DSS and F-AOM/DSS groups, implicating the Nrf2-related antioxidant reaction as an underlying mechanism of the protective effect of estradiol. The high expression levels of Nrf2 and its related anti-oxidant enzyme in the M-AOM/DSS+estr group further strengthen this suggestion.

There was an increase of the NLRP3 inflammasome and its effector cytokines (IL-1 $\beta$ and IL-18) simultaneously with increased Nrf2 on the DSS-induced inflammation stage. Considering the importance of Nrf2 in NLRP3 inflammasome activation [15], Nrf2 activation might lead to immune modulation through caspase-1 related activities, such as pyroptosis [16]. Based on the knowledge that NLRP3 inflammasome activation induces pyroptosis [16], we hypothesize that Nrf2/NLRP3 inflammasome/IL-1 $\beta$-mediated pyroptosis triggers the elimination of precancerous cells during inflammation, and further prevents carcinogenesis in the presence of estradiol (Fig. 5A). To prove this hypothesis, further investigations are required.

Since estradiol administration completely inhibited inflammation in AOM/DSS-treated male mice resulting in the nearcomplete prevention of CRC, we expected that AOM/DSS- 
induced inflammation at week 2 would be mild in female mice. There was a significant difference of DAI at week 2 between male and female mice, but not such significant differences in the severity of inflammation reflected in COX-2. The inflammation represented by DAI cannot be fully explained by a few inflammatory mediators, such as COX-2. The strong effect of administered estradiol on preventing AOM/ DSS-induced inflammation and colon tumorigenesis might be due to the higher concentration of intraperitoneal-administered estradiol; the injection concentration was $10 \mathrm{mg} / \mathrm{kg}$, compared to the concentration of endogenous estradiol of 4 $\mathrm{pg} / \mathrm{mL}$ in female mice [27]. Furthermore, the endogenous estradiol level in mice sacrificed at week 2 might be lower than that of fully developed female mice. Also, sex difference in stress susceptibility may affect levels of some pro-inflammatory cytokines (e.g., IL-1ß) [28], implying that endogenous estradiol has more complex action than exogenously injected estradiol. However, in terms of cancer prevention, as shown in S4 Fig. that compares the F-AOM/DSS non-cancer group with the F-AOM/DSS cancer group, endogenous estradiol seems to have a similar effect and mechanism, including PKC $\delta$ and inflammasome with exogenous estradiol. Different methods of estradiol treatment, various estradiol concentrations, and blood estradiol level monitoring can be considered to provide more physiologic conditions. To further investigate the effect of endogenous estradiol and its underlying mechanism, experiments using the ovariectomized female mice are underway.

After tumors developed (weeks 10 and 16), we observed the interesting finding that Nrf2 signaling was significantly up-regulated in the M-AOM/DSS group, suggesting that Nrf2 and anti-oxidant enzymes might play a role in promoting tumor progression (Fig. 5B). Several studies reported the possibility of the dual role of Nrf2 in tumor prevention and progression [29]. Satoh et al. [29] showed that Nrf2 activation in cancer cells enhances tumor malignancy, while Keap1knockdown mice having high expression of Nrf2 are more resistant to urethane-induced carcinogenesis. Similar to Nrf2, we found two facets of NLRP3 inflammasome. Although NLRP3 inflammasome is a well-established target of NF- $\mathrm{kB}$, its expression is inversely related to NF- $\mathrm{KB}$ expression at week 2 . This indicates that it might have different roles from $\mathrm{NF}-\mathrm{\kappa B}$, such as inducing pyroptosis in the DSS-induced inflammation stage. In contrast, it has been reported that once tumor formation is initiated, NLRP3 inflammasomeinduced IL-1 $\beta$ and IL-18 modulate immunity in the tumor microenvironment, and promote cancer progression [30]. A significant increment of NLRP3 and caspase-1 expression in the F-AOM/DSS cancer group compared to the F-AOM/DSS non-cancer group in the present study also supports tumor promotion by the NLRP3 inflammasome.

The collective present and prior $[8,16,22]$ data support the proposal that the regulatory mechanism of estradiol in colitis-associated CRC depends on sex, and the timing of DSSinduced inflammation and carcinogenesis (Fig. 6). At the peak of inflammation at week 2, estradiol appears to induce inflammasome activation through $\mathrm{G} \alpha_{13}$ protein subunits. $G \alpha_{12}$ and $\mathrm{G} \alpha_{13}$ have potentiated estradiol-bound ER $\alpha$ activity [22]. However, despite the functional overlap between $G \alpha_{12}$ and $\mathrm{G} \alpha_{13}$, only $\mathrm{G} \alpha_{13}$ regulates NRF2 via PKC $\delta$ [8]. NRF2 mediates inflammasome activation through the transcription of as-yet unknown genes. NLRP3 inflammasome activation induces pyroptosis to eliminate precancerous cells [16]. NRF2 inhibits NF- $\kappa \mathrm{B}$, which is activated by inflammatory activators through Toll-like receptor signaling and reactive oxygen species. Ultimately, estradiol prevents carcinogenesis, whereas in the absence of estradiol, a cancer inducing microenvironment is created through NF-kB activation. When precancerous cells are not completely eliminated, cancer progresses through both the $G \alpha_{12}$ and $G \alpha_{13}$ protein subunits. $G \alpha_{12}$ regulates the NF- $\kappa B$ mediated signaling pathway $[8,23]$. $G \alpha_{13}$ regulates NRF2 via PKC $\delta$ [8]. NRF2 promotes tumor progression by the activation of anti-oxidant enzymes and NLRP3 inflammasome [15]. Ultimately, NF-kB and NRF2 signaling pathways accelerate carcinogenesis.

In conclusion, our study shows estradiol administration in $\mathrm{AOM} / \mathrm{DSS}$-treated male mice attenuates inflammation, and increases Nrf2 in the DSS-induced inflammation stage. Moreover, inhibition of the NF-kB-related pathway and activation of Nrf2-related anti-oxidant enzymes and the NLRP3 inflammasome pathway indicate possible underlying mechanisms. This study finally suggests that Nrf2 and the NLRP3 inflammasome play a dual role, with a preventive effect on tumor development, but promotion of tumor progression, once a tumor is initiated.

\section{Electronic Supplementary Material}

Supplementary materials are available at Cancer Research and Treatment website (https://www.e-crt.org).

\section{Conflicts of Interest}

Conflict of interest relevant to this article was not reported.

\section{Acknowledgments}

This work was supported by a grant from the National Research Foundation of Korea (NRF) funded by the government of the Republic of Korea (2016R1A2B4013133). 


\section{References}

1. Alteri R, Kramer J, Simpson S. Colorectal cancer facts and figures 2014-2016. Atlanta, GA: American Cancer Society; 2014.

2. Gierisch JM, Coeytaux RR, Urrutia RP, Havrilesky LJ, Moorman PG, Lowery WJ, et al. Oral contraceptive use and risk of breast, cervical, colorectal, and endometrial cancers: a systematic review. Cancer Epidemiol Biomarkers Prev. 2013;22:1931-43.

3. Chlebowski RT, Wactawski-Wende J, Ritenbaugh C, Hubbell FA, Ascensao J, Rodabough RJ, et al. Estrogen plus progestin and colorectal cancer in postmenopausal women. N Engl J Med. 2004;350:991-1004.

4. Weyant MJ, Carothers AM, Mahmoud NN, Bradlow HL, Remotti H, Bilinski RT, et al. Reciprocal expression of ERalpha and ERbeta is associated with estrogen-mediated modulation of intestinal tumorigenesis. Cancer Res. 2001;61:2547-51.

5. Amos-Landgraf JM, Heijmans J, Wielenga MC, Dunkin E, Krentz KJ, Clipson L, et al. Sex disparity in colonic adenomagenesis involves promotion by male hormones, not protection by female hormones. Proc Natl Acad Sci U S A. 2014;111: 16514-9.

6. Lee SM, Kim N, Son HJ, Park JH, Nam RH, Ham MH, et al. The effect of sex on the azoxymethane/dextran sulfate sodium-treated mice model of colon cancer. J Cancer Prev. 2016;21: 271-8.

7. Wu J, Williams D, Walter GA, Thompson WE, Sidell N. Estrogen increases Nrf2 activity through activation of the PI3K pathway in MCF-7 breast cancer cells. Exp Cell Res. 2014;328: 351-60.

8. Cho MK, Kim WD, Ki SH, Hwang JI, Choi S, Lee CH, et al. Role of Galpha12 and Galpha13 as novel switches for the activity of Nrf2, a key antioxidative transcription factor. Mol Cell Biol. 2007;27:6195-208.

9. Li T, Xiao X, Zhang J, Zhu Y, Hu Y, Zang J, et al. Age and sex differences in vascular responsiveness in healthy and trauma patients: contribution of estrogen receptor-mediated Rho kinase and PKC pathways. Am J Physiol Heart Circ Physiol. 2014;306:H1105-15.

10. Wang Y, Zhu L, Kuokkanen S, Pollard JW. Activation of protein synthesis in mouse uterine epithelial cells by estradiol17beta is mediated by a PKC-ERK1/2-mTOR signaling pathway. Proc Natl Acad Sci U S A. 2015;112:E1382-91.

11. O'Mahony F, Alzamora R, Chung HL, Thomas W, Harvey BJ. Genomic priming of the antisecretory response to estrogen in rat distal colon throughout the estrous cycle. Mol Endocrinol. 2009;23:1885-99.

12. Khor TO, Huang MT, Prawan A, Liu Y, Hao X, Yu S, et al. Increased susceptibility of Nrf2 knockout mice to colitis-associated colorectal cancer. Cancer Prev Res (Phila). 2008;1:18791.

13. Li W, Khor TO, Xu C, Shen G, Jeong WS, Yu S, et al. Activation of Nrf2-antioxidant signaling attenuates NFkappaB-inflammatory response and elicits apoptosis. Biochem Pharmacol. 2008;76:1485-9.

14. Niture SK, Khatri R, Jaiswal AK. Regulation of Nrf2-an update. Free Radic Biol Med. 2014;66:36-44.

15. Zhao C, Gillette DD, Li X, Zhang Z, Wen H. Nuclear factor
E2-related factor-2 (Nrf2) is required for NLRP3 and AIM2 inflammasome activation. J Biol Chem. 2014;289:17020-9.

16. Miao EA, Rajan JV, Aderem A. Caspase-1-induced pyroptotic cell death. Immunol Rev. 2011;243:206-14.

17. Kepp O, Galluzzi L, Zitvogel L, Kroemer G. Pyroptosis: a cell death modality of its kind? Eur J Immunol. 2010;40:627-30.

18. Saleiro D, Murillo G, Benya RV, Bissonnette M, Hart J, Mehta RG. Estrogen receptor-beta protects against colitis-associated neoplasia in mice. Int J Cancer. 2012;131:2553-61.

19. Choi YJ, Choi YJ, Kim N, Nam RH, Lee S, Lee HS, et al. Acai berries inhibit colon tumorigenesis in azoxymethane/dextran sulfate sodium-treated mice. Gut Liver. 2017;11:243-52.

20. Suzuki R, Kohno H, Sugie S, Tanaka T. Sequential observations on the occurrence of preneoplastic and neoplastic lesions in mouse colon treated with azoxymethane and dextran sodium sulfate. Cancer Sci. 2004;95:721-7.

21. Lee SE, Yang H, Jeong SI, Jin YH, Park CS, Park YS. Induction of heme oxygenase-1 inhibits cell death in crotonaldehydestimulated HepG2 cells via the PKC-delta-p38-Nrf2 pathway. PLoS One. 2012;7:e41676.

22. Bratton MR, Antoon JW, Duong BN, Frigo DE, Tilghman S, Collins-Burow BM, et al. Galphao potentiates estrogen receptor alpha activity via the ERK signaling pathway. J Endocrinol. 2012;214:45-54.

23. Ki SH, Choi MJ, Lee CH, Kim SG. Galpha12 specifically regulates COX-2 induction by sphingosine 1-phosphate. Role for JNK-dependent ubiquitination and degradation of IkappaBalpha. J Biol Chem. 2007;282:1938-47.

24. DiDonato JA, Mercurio F, Karin M. NF-kappaB and the link between inflammation and cancer. Immunol Rev. 2012;246: 379-400.

25. Kalaitzidis D, Gilmore TD. Transcription factor cross-talk: the estrogen receptor and NF-kappaB. Trends Endocrinol Metab. 2005;16:46-52.

26. Kim W, Lee HN, Jang JH, Kim SH, Lee YH, Hahn YI, et al. 15-Deoxy-delta(12,14)-prostaglandin J2 exerts proresolving effects through nuclear factor E2-related factor 2-induced expression of CD36 and heme oxygenase-1. Antioxid Redox Signal. 2017;27:1412-31.

27. Haisenleder DJ, Schoenfelder AH, Marcinko ES, Geddis LM, Marshall JC. Estimation of estradiol in mouse serum samples: evaluation of commercial estradiol immunoassays. Endocrinology. 2011;152:4443-7.

28. Lee JY, Kim N, Kim YS, Nam RH, Ham MH, Lee HS, et al. Repeated water avoidance stress alters mucosal mast cell counts, interleukin-1beta levels with sex differences in the distal colon of Wistar rats. J Neurogastroenterol Motil. 2016;22: 694-704.

29. Satoh H, Moriguchi T, Saigusa D, Baird L, Yu L, Rokutan H, et al. NRF2 intensifies host defense systems to prevent lung carcinogenesis, but after tumor initiation accelerates malignant cell growth. Cancer Res. 2016;76:3088-96.

30. Fabbi M, Carbotti G, Ferrini S. Context-dependent role of IL-18 in cancer biology and counter-regulation by IL-18BP. J Leukoc Biol. 2015;97:665-75. 